\title{
Making Aircraft Vortices Visible to Radar by Spraying Water into the Wake $\mathscr{A}^{\mathscr{A}}$
}

\author{
KARIM SHARIFF \\ NASA Ames Research Center, Moffett Field, California
}

(Manuscript received 31 March 2016, in final form 1 August 2016)

\begin{abstract}
Aircraft trailing vortices pose a danger to following aircraft during takeoff and landing. This necessitates spacing rules, based on aircraft type, to be enforced during approach in instrument flight regulation (IFR) conditions; this can limit airport capacity. To help choose aircraft spacing based on the actual location and strength of the wake, it is proposed that wake vortices can be detected using conventional ground-based precipitation and cloud radars. This is enabled by spraying a small quantity of water into the wake from near the wing. The vortex strength is revealed by the Doppler velocity of the droplets. In the present work, droplet size distributions produced by nozzles used for aerial spraying are considered. Droplet trajectory and evaporation in the flow field is numerically calculated for a heavy aircraft, followed by an evaluation of radar reflectivity at 6 nautical miles $(\mathrm{nmi})$ behind the aircraft. Small droplets evaporate, while larger droplets fall out of the wake. In the humid conditions that typically prevail during IFR, a sufficient number of droplets remain in the wake and give good signal-to-noise ratios (SNR). For conditions of average humidity, higherfrequency radars combined with spectral processing give good SNR.
\end{abstract}

\section{Introduction}

\section{a. Motivation}

Aircraft trailing vortices are a hazard to following airplanes during takeoff and landing (e.g., Barbagallo 2014). For a review of their dynamics, see Spalart (1998). Because of their mutually induced velocity, a pair of trailing vortices generally descends from the altitude where it was generated [for exceptions to this in strongly stratified conditions, see Spalart (1996)]. Therefore, a wake encounter may occur when a following aircraft finds itself below ${ }^{1}$ the path of the leading aircraft. The possibility for this is increased during takeoff and

\footnotetext{
${ }^{1}$ A former colleague, Dr. Vernon Rossow, has long suggested that with GPS and fly-by-wire controls, the simplest approach to wake avoidance would be for a following aircraft to remain at or above the path of the leader.
}

Supplemental information related to this paper is available at the Journals Online website: http://dx.doi.org/10.1175/ JTECH-D-16-0066.s1.

Corresponding author address: Karim Shariff, NASA Ames Research Center, Mail Stop 258-1, Moffett Field, CA 94035.

E-mail: karim.shariff@nasa.gov landing, and the present work addresses the latter situation. When cleared for a visual approach to landing, the pilot of the following aircraft can visually attempt to remain above the path of the leader by, for example, flying at a higher glide slope to the same touchdown point as the leader. Even in visual approaches, however, things can and do go awry (Barbagallo 2014, section 2.3). This means that efforts to find an all-weather wake sensor should be continued.

When the ceiling is less than $1000 \mathrm{ft}$ and the visibility is less than 3 statute miles, operations must be conducted under instrument flight regulations (IFR). In this case, air traffic controllers maintain separations according to the weight categories of the leading and following aircraft; see Table 1 . These separations have started to limit capacity at some airports (Crouch et al. 2001b) and we refer the reader to National Research Council (2008). About two decades ago, NASA initiated the Aircraft Vortex Spacing System (AVOSS) program, whose aim was to make aircraft spacing dynamic through a combination of vortex sensing and real-time flowfield simulation. The present work is motivated by the vortex detection aspect of the AVOSS program. A current effort that has similar aims as the defunct AVOSS program is WakeNet-Europe (www.wakenet.eu). Finally, we mention the development of a system to hasten the breakup of trailing vortices by exciting vortex instabilities 
TABLE 1. IFR separation standards ( $\mathrm{n} \mathrm{mi)} \mathrm{for} \mathrm{arrivals} \mathrm{on} \mathrm{the} \mathrm{same}$ runway (Barbagallo 2014).

\begin{tabular}{lccccc}
\hline \hline & \multicolumn{5}{c}{ Follower } \\
\cline { 2 - 6 } \multicolumn{1}{c}{ Leader } & Super & Heavy & Boeing 757 & Large & Small \\
\hline Super & 3 & 6 & 7 & 7 & 8 \\
Heavy & 3 & 4 & 5 & 5 & 6 \\
Boeing 757 & 3 & 4 & 4 & 4 & 5 \\
Large & 3 & 3 & 3 & 3 & 4 \\
Small & 3 & 3 & 3 & 3 & 3 \\
\hline
\end{tabular}

through periodic motion of control surfaces (Crouch et al. 2001a,b). Such a system could be implemented together with one for wake detection.

\section{b. Previous work using radar to detect aircraft wakes}

The main vortex detection technology tested by the AVOSS program was infrared ground-based lidar. A concern raised about lidar was that since water vapor strongly absorbs infrared, it would not be usable in IFR conditions. Another concern is that optical systems are more expensive and difficult to maintain than radar. These concerns motivate consideration of radar. The next two subsections discuss the two contexts in which previous work on the radar reflectivity of aircraft wakes has been performed, namely, clear-air reflectivity and the exploitation of natural precipitation; for a recent review see Barbaresco et al. (2016).

\section{1) Clear-Air Reflectivity}

The first observational and theoretical efforts on ground-based radar detection of wakes were in the context of clear air. Systematic tests by Gilson (1992) showed that the wake of a C-5A aircraft could be detected by radars having 2-7 MW of peak power at frequencies from 0.162 to $5.67 \mathrm{GHz}$, with no return at $35 \mathrm{GHz}$. Shariff and Wray (2002) analyzed the reflectivity in Gilson's test using a model of a vortex pair descending in a stratified atmosphere, carrying with it an oval of atmospheric air from the altitude at which the wake formed. This leads to a gradient in refractive index between the oval and ambient air. Another mechanism investigated by Shariff and Wray (2002), which has peak reflectivity at a low frequency of $50 \mathrm{MHz}$, is the pressure (hence density) gradient in each vortex. Both of these mechanisms have some drawbacks for practical use: (i) the radar cross section is small $\left(-60\right.$ to $\left.-80 \mathrm{~dB} \mathrm{~m}^{2}\right)$; (ii) the first mechanism depends on atmospheric stratification, which has seasonal, geographic, and diurnal variations; and (iii) the frequency of the second mechanism is the same as that of stratospheric-tropospheric radars, which have an antenna array on the ground looking upward. For the present application, technology would have to be developed for aiming the radar by use of phasing. Li et al. (2011) (see also Vanhoenacker-Janvier et al. 2012) extended the work of Shariff and Wray (2002) with a better calculation of the compressibilityinduced variation in air density in the wake. More importantly, they showed that the atmospheric gradient of water vapor is wound up into a spiral by each vortex, which allows scattering at high frequencies. Unfortunately, radar reflectivity is not studied beyond an evolution time of $40 \mathrm{~s}$, which corresponds to $1.7 \mathrm{n}$ mi behind the aircraft.

Barbaresco et al. (2008) conducted tests using an $\mathrm{X}$-band $(9.6 \mathrm{GHz})$ radar for aircraft taking off and flying straight and level at $1500 \mathrm{~m}$ altitude. For the takeoff case, the range was about $700 \mathrm{~m}$ looking roughly sideways using peak pulse power of only $20 \mathrm{~W}$. For the aircraft at an altitude of $1500 \mathrm{~m}$, the radar was looking straight up and the peak pulse power was $75 \mathrm{~W}$. A timeDoppler plot indicated a spiral structure in each vortex. However, in staring mode, a return is obtained for only $5 \mathrm{~s}$, at most. Barbaresco et al. (2008) did not report how far downstream of the aircraft the wake could be detected; it appears that this distance is very short.

In conclusion, clear-air reflectivity is an interesting prospect, but more careful and better documented observational campaigns combined with theoretical efforts are needed.

\section{2) EXPLOITING NATURAL PRECIPITATION}

A different approach that the author first heard about from Robert Neece (NASA Langley Research Center, 2000, personal communication) exploits the fact that water droplets (in the form of fog or rain) are present in IFR weather conditions. They are strong radar reflectors, and, when an airplane wake sets these particles into motion, they can be separated from ambient droplets via their Doppler signature. This strategy has the advantage that standard Doppler weather radars could be used. Seliga and Mead (2009) demonstrated the feasibility of the approach using a W-band $(94 \mathrm{GHz})$ radar in light rain in an opportunistic test with only $100 \mathrm{~mW}$ of peak power. An analysis of the reflectivity of this mechanism has been conducted by Liu et al. (2013) and $\mathrm{Li}$ et al. (2016) for rain and fog, respectively. In spring 2011, successful measurements (Barbaresco et al. 2012) in rain were obtained at Charles De Gaulle Airport in Paris, France, using a Thales BOR-A 550 X-band radar with only $20 \mathrm{~W}$ of peak power; see Barbaresco (2012) for more legible figures. The results of a campaign (also in rain) during fall 2012 are reported in Barbaresco et al. (2014), which presents plots at different times in three dimensions. The main drawback of this approach is that there may be long periods of time, and stretches 
along the wake, during which a sufficient number of natural droplets are unavailable.

\section{c. Present approach and concept of operations}

The approach proposed here is related to the use of natural precipitation for wake detection discussed in the previous paragraph, except that, to provide a persistent radar target, water spray is injected into the wake. We envision one nozzle on each side of the airplane that injects the water spray near the wing trailing edge at a specified spanwise location. One possibility for nozzle placement is the aft tip of a flap track fairing. A pump and a water tank could be located nearby within the wing.

The present work considers a single ground-based radar, placed between the two outermost approach paths of several parallel runways in order that it can monitor all approach paths. The choice of radar coordinates is described in detail in section $2 \mathrm{~m}$. Most of the results presented are for the radar looking (with normal incidence) at the wake cross section $6 \mathrm{n}$ mi behind the aircraft. The radar can also perform an azimuthal scan to monitor several wake cross sections along the flight paths; see section $3 i$. However, the off-normal cross sections have a reduced SNR due to increased range and a reduction in Doppler velocity by the cosine of the angle relative to the normal. Given the successful detection of a wake, pilots could be presented with that information on a heads-up display and be cleared for an approach using that information.

The main constraint imposed by nature is droplet retention in the wake: a spray nozzle produces a distribution of droplet sizes; the smallest droplets evaporate, while the largest ones fall out of the wake due to gravity. However, IFR conditions are correlated with high humidity (section 21). This reduces the rate of evaporation and makes the approach feasible. In a non-IFR case we consider to have moderate humidity, only the Ka- and $\mathrm{W}$-band radars give sufficient reflectivity in a patch above each vortex. However, it is shown that spectral processing reduces noise and allows detection even when the signal-to-noise ratio in individual pulse returns is less than 1. Finally, there is no reason why the present approach and that of using natural precipitation could not be combined using the same radar. It should be noted that spray droplets can also be detected using lidar: they would enhance the signal-to-noise ratio over natural aerosols and therefore reduce the required power.

\section{Calculation methods}

\section{a. General procedure}

We use aircraft centered coordinates $(x, y, z)$, where $x$ is streamwise, $y$ is spanwise, and $z$ is vertical. Air and water are denoted by subscripts $a$ and $w$, respectively.
The calculation has three steps. The first creates a sample of droplet radii $a(t)$ from a size distribution pertinent to aerial spray nozzles. The second step generates a spray trail on the starboard side of the aircraft wake. This involves injecting droplets from the sample into the wake, tracking the position $\mathbf{X}(t)$ of each droplet and $a(t)$ as it evaporates. The flow-field model consists of two counterrotating vortices whose height decreases with downstream distance $x$ behind the aircraft. The third and final step mirrors the starboard trail to the port side and, for a given set of radar parameters, computes the reflectivity of both trails together.

Since it is prohibitive to track all of the actual droplets, the spray trail computed in step 2 consists of a certain number, $N_{\text {comp }}$, of computational droplets. In the reflectivity calculation, each computational droplet is taken to represent a multiplicity, $M_{\text {actual }}$, of actual droplets, which is simply the ratio of the desired injected volume to the volume injected in the computation. A test showing that statistical convergence has been achieved is presented in section 3e.

The equations for droplet motion and evaporation are integrated using the routine Livermore Solver for Ordinary Differential Equations (LSODE), which is described in Radhakrishnan and Hindmarsh (1993) and available from Netlib. The routine chooses a time step based on a specified error tolerance. Since the flow-field model we have adopted is steady in a reference frame moving with the aircraft, it is not necessary to inject droplets at every time step, which would continuously increase the number of computational droplets. Instead, droplets are injected only during a certain time interval $\Delta t_{0}$ at the beginning of the calculation. This set of droplets is then evolved for successive $\Delta t_{0}$ intervals and appended to a file at the end of every $\Delta t_{0}$ interval. At the end of the computation, the file contains a spray trail that is $7 \mathrm{nmi}$ long, whose radar reflectivity we subsequently analyze. In actual practice, an aircraft would likely not generate a trail of several nautical miles behind it. Rather, it would release the spray for a short period at preselected locations during its approach.

A detailed description of each part of the procedure is described in the following subsections. The evaporation calculation is described in section B of the online supplemental material. To avoid stiffness of the system of evolution equations, droplets are removed from the calculation when their radius becomes smaller than $20 \mu \mathrm{m}$; at this point their reflectivity is too small to significantly affect the received power.

\section{b. Droplet trajectory}

The position $\mathbf{X}(t)$ and velocity $\mathbf{U}(t)$ of a droplet of mass $m_{d}$ evolves according to 


$$
\begin{aligned}
& \frac{d \mathbf{X}}{d t}=\mathbf{U}(t), \\
& \frac{d \mathbf{U}}{d t}=\mathbf{F}_{D} / m_{d}-g_{\mathrm{eff}} \hat{\mathbf{z}},
\end{aligned}
$$

where $g_{\text {eff }}=\left(1-\rho_{a} / \rho_{w}\right) g$ is the effective gravity accounting for buoyancy and $\hat{\mathbf{z}}$ is the unit vector in the vertical direction. The drag force $\mathbf{F}_{D}$ is given by

$$
\mathbf{F}_{D}=C_{D} \frac{1}{2} \rho_{a}\left|\mathbf{u}_{\text {rel }}\right|^{2} \pi a^{2} \frac{\mathbf{u}_{\text {rel }}}{\left|\mathbf{u}_{\text {rel }}\right|},
$$

where

$$
\mathbf{u}_{\text {rel }}=\mathbf{u}(\mathbf{X})-\mathbf{U}
$$

is the velocity of the airflow, $\mathbf{u}(\mathbf{X})$, relative to the droplet. Evaluation of the drag coefficient, $C_{D}$, is described in section $\mathrm{A}$ of the online supplemental material.

\section{c. Droplet size distribution of aerial spray nozzles}

When the water jet issues from the nozzle, it will encounter a blast of free-stream air with a speed of $77.2 \mathrm{~m} \mathrm{~s}^{-1}$. A comparable situation in the literature is that of a cylindrical liquid jet surrounded by an annulus of coflowing air (Lorenzetto and Lefebvre 1977; Varga et al. 2003), which shows that much smaller droplets are produced than for the case of still air (with the same velocity of the liquid jet). This is due to the occurrence of both the Kelvin-Helmholtz instability and Rayleigh-Taylor instabilities. The former is driven by the shear between the air and liquid flow and leads to a smaller instability wavelength. The latter arises due to the acceleration of liquid droplets by the drag force of the airstream. If the droplets are too small, they quickly evaporate. To produce larger droplets, both instabilities can be mitigated by reducing the relative velocity between the liquid jet and freestream air. This is accomplished by increasing the driving pressure. However, if the droplets are too large, they fall out of the wake. Hence, there is an optimum droplet size.

We were fortunate that an experimental study (Fritz and Hoffmann 2015) that uses a wind tunnel to mimic the free-stream airflow has recently been performed to characterize the droplet size distribution produced by nozzles used for aerial agricultural spraying. This study did indeed show that larger pressures produce larger droplets. Dr. Brad Fritz (USDA) kindly provided us with an Excel program, developed from that study, that gives parameters of the droplet size distribution for various nozzles, free-stream speeds, and driving pressures. Use of these parameters is now described.

Let $p(a)$ be the probability density such that $p(a) d a$ is the probability that the droplet radius is in the interval $[a, a+d a]$. The lognormal distribution

$$
p(a)=\frac{1}{\sqrt{2 \pi} a \sigma} e^{-\ln ^{2}\left(a / a_{0}\right) / 2 \sigma^{2}},
$$

with parameters $a_{0}$ and $\sigma$, is commonly used in the spray literature. Fritz's Excel program provides information about the function $Q(a)$, defined to be the fractional volume occupied by droplets of radius $\leq a$. One can show from appropriately integrating (5) that

$$
Q(a)=\frac{1}{2}(1+\operatorname{erf} \xi)
$$

where

$$
\xi \equiv \frac{1}{\sqrt{2} \sigma}\left[\ln \left(a / a_{0}\right)-3 \sigma^{2}\right]
$$

The Excel program provides $a_{0.5}$ and $a_{0.9}$ defined such that $Q\left(a_{0.5}\right)=0.5$ and $Q\left(a_{0.9}\right)=0.9$. Using them, (6) can be numerically inverted to yield the parameters $a_{0}$ and $\sigma$ of the lognormal distribution [(5)]. The Excel program also provides $a_{0.1}$, which we did not use because we wished to nail the size distribution for large droplets, which contribute most to reflectivity.

\section{d. Choice of nozzle}

What is the best drop size distribution? A set of $N_{d}$ droplets over which the incident beam is assumed to have uniform intensity has a reflectivity proportional to (e.g., Doviak and Zrnić 1984, p. 58)

$$
\zeta=\sum_{i=1}^{N_{d}} a_{i}^{6},
$$

assuming Rayleigh scattering. Maximizing this subject to a fixed volume of water and a fixed $N_{d}$ gives the result that all droplets must be of the same size. Given this result, maximizing $\zeta$ with respect to the number $N_{d}$ subject to fixed volume gives $N_{d}=1$; that is, all the volume must be in one droplet. However, such a droplet would likely fall too rapidly. To minimize droplet loss by sedimentation, droplets must not have a terminal velocity larger than the vortex descent speed, $W_{\text {descent }}=1.75 \mathrm{~m} \mathrm{~s}^{-1}$ in the present case. Consulting the terminal velocity plot in Pruppacher and Klett (1997, p. 416), we conclude that $a$ must be $\leq 200 \mu \mathrm{m}$. Hence, the best distribution is uniform with a drop radius of $a=200 \mu \mathrm{m}$. Since the rate of droplet evaporation is $\propto 1 / a$-that is, small droplets evaporate faster than larger ones- the above-mentioned conclusion is not altered by including evaporation.

The above-mentioned considerations suggest that the following "rate of $\zeta$ " could be used to initially evaluate different nozzles without having to perform an evaporation and reflectivity calculation, 
TABLE 2. Aerial nozzle parameters for the operating conditions specified. "CP" denotes CP Products.

\begin{tabular}{|c|c|c|c|c|}
\hline & Nozzle 1 & Nozzle 2 & Nozzle 3 & Nozzle 4 \\
\hline Model & CP-09 & CP-09 & CP11TT & Davidon Tri-Set \\
\hline Pressure (psi) & 90 & 90 & 90 & 90 \\
\hline Airspeed (mph) & 175 & 175 & 175 & 175 \\
\hline Deflection plane/body angle & $0^{\circ}$ & $0^{\circ}$ & $0^{\circ}$ & $0^{\circ}$ \\
\hline Fan angle $^{\mathrm{a}}$ & Str & Str & Str & Str \\
\hline Orifice code & & & 20 & \\
\hline Orifice diameter (in.) & 0.125 & 0.172 & 0.105 & 0.125 \\
\hline$a_{0.5}(\mu \mathrm{m})^{\mathrm{b}}$ & 178.5 & 146.5 & 183 & 239.5 \\
\hline$a_{0.9}(\mu \mathrm{m})^{\mathrm{b}}$ & 315 & 303 & 359 & 463 \\
\hline$a_{0}$ of lognormal & 99.02 & 55.83 & 79.85 & 108.3 \\
\hline$\sigma$ of lognormal & 0.443 & 0.567 & 0.526 & 0.514 \\
\hline Flow rate $(\mathrm{gpm})^{\mathrm{c}}$ & 3.70 & 6.49 & 3.00 & 3.06 \\
\hline$U_{\text {exit }}\left(\mathrm{m} \mathrm{s}^{-1}\right)$ & 29.5 & 27.3 & 33.9 & 24.4 \\
\hline No. of nozzles per side & 1 & 1 & 1 & 1 \\
\hline Gallons per $\mathrm{n}$ mi (two sides) & 2.96 & 5.19 & 2.4 & 2.45 \\
\hline
\end{tabular}

a "Str" denotes a straight stream.

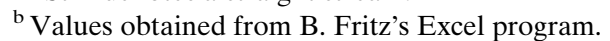

${ }^{\mathrm{c}}$ See text for how flow rates were obtained.

$$
\dot{\zeta}_{\text {nozzle }}^{<} \equiv \frac{1}{\Delta t} \sum_{i: a_{i}<200 \mu \mathrm{m}}^{N_{d}(\Delta t)} a_{i}^{6}
$$

where the less than superscript $(<)$ on $\dot{\zeta}$ denotes the exclusion from the sum of large droplets that fall away, and $N_{d}(\Delta t)$ denotes the number of droplets produced by the nozzle in a period $\Delta t$. The usefulness of $\dot{\zeta}_{\text {nozzle }}^{<}$as a simple a priori measure will be tested against the full reflectivity calculations in section $3 \mathrm{~h}$.

Table 2 lists the parameters for four candidate nozzles and operating conditions that were selected from Fritz's Excel program. The nozzles were selected based on having a peak probability density at a large radius (which rarely exceeded $100 \mu \mathrm{m}$ ) and a high flow rate. The flow rate for nozzle 1 was provided by Calvin Kroes (CP Products, 2015, private communication). For nozzles 2 and 3, flow rates were extrapolated from the values of $5.30 \mathrm{gpm}$ and $2.45 \mathrm{gpm}$, respectively, at $60 \mathrm{psi}$ as reported on the manufacturer's data sheets (www. translandllc.com/wp-content/uploads/2015/08/Aerial-FlowChart-20152.pdf; www.cpproductsinc.com/images/stories/ downloads/Misc-Tables/A1-Web\%20Aerial\%20Tip\% 20Rate $\% 20$ Chart.pdf). The extrapolations assumed a square root dependence of flow rate on pressure (Lefebvre 1989 , p. 157) as expected from Bernoulli's principle, and which the manufacturer's data follow well. For nozzle 4 , the flow rate versus pressure provided on the manufacturer's website has a linear dependence rather than a square root dependence. We are grateful to Fritz for measuring the actual flow rate for us. It turned out to be much lower than the value provided on the manufacturer's website.

\section{e. Droplet injection in the computation}

Let the origin of coordinates be in the symmetry plane of the aircraft (which corresponds to $y=0$ ) with the same axial and vertical location as the droplet injector. Droplets are injected in a grid pattern within a square of width $w_{\text {square }}$ in the $x-y$ plane. The pattern consists of $n_{\text {square }} \times n_{\text {square }}$ droplets. The square is centered at $(x, y, z)=(0, f b / 2,0)$, where $f$ represents the fractional spanwise distance from the aircraft center plane to the wingtip and was chosen to be $f=0.5$. A square shape was chosen because the nozzles we have selected are not of the flat fan type. The streamwise extent of the computed mist trail was chosen to be $\ell_{\text {trail }}=7 \mathrm{n} \mathrm{mi}(168 \mathrm{~s}$ of elapsed time from injection), since we wish to detect the trail at $6 \mathrm{~nm}$, the longest distance for which one would want to detect the wake of a heavy aircraft under current separation rules; see Table 1.

The square pattern is injected $n_{x}$ times and the time interval between injections is $\Delta t_{\text {inject }}$, whose value is chosen so that the $x$ spacing between droplets is the same as in the cross-sectional $(y-z)$ plane. After a period $\Delta t_{0} \equiv n_{x} \Delta t_{\text {inject }}$, an $n_{\text {square }}^{2} \times n_{x}$ slab of particles has been injected, which is then advanced for successive $\Delta t_{0}$ periods to form the entire trail. Inertial particles with a small Stokes number tend to an attractor (Haller and Sapsis 2008; Sapsis and Haller 2010) independent of injection location, and therefore where droplets end up should be insensitive to where they are injected. A brief check on insensitivity to initial conditions will be presented in section $3 \mathrm{c}$.

The initial velocity of droplets is set equal to the air velocity, which is justified as follows. From the equations 
TABLE 3. Parameters of a typical heavy aircraft. Approach speed in knots $\left(\mathrm{kt} ; 1 \mathrm{kt}=0.51 \mathrm{~m} \mathrm{~s}^{-1}\right)$.

\begin{tabular}{lc}
\hline \hline \multicolumn{1}{c}{ Parameter } & Value \\
\hline Weight, $W$ & $500000 \mathrm{lb}$ \\
Wing span, $b$ & $60 \mathrm{~m}$ \\
Vortex spacing, $b_{0}$ & $47.9 \mathrm{~m}$ \\
Vortex circulation, $\Gamma$ & $526 \mathrm{~m}^{2} \mathrm{~s}^{-1}$ \\
Approach speed, $U_{\text {app }}$ & $150 \mathrm{kt}$ \\
\hline
\end{tabular}

of droplet motion [(2)-(4)], the characteristic relaxation time for a droplet to start following a new airspeed, imposed at $t=0$, say, is

$$
\tau_{\text {relax }} \equiv\left|\frac{1}{u_{\text {rel }}} \frac{d u_{\text {rel }}}{d t}\right|_{0}^{-1}=\frac{16}{3} \frac{\rho_{w}}{\rho_{a}} \frac{a^{2}}{\nu_{a}}\left(\operatorname{Re} C_{D}\right)_{0}^{-1},
$$

where the subscript " 0 " means that the quantity is calculated at $t=0$. From this, the characteristic relaxation distance $\ell_{\text {relax }}=u_{\text {rel }}(0) \tau_{\text {relax }}$ can be evaluated. Note that the initial airspeed relative to the water jet is given by $u_{\text {rel }}(0)=U_{\text {app }}-U_{\text {exit }}$, where $U_{\text {app }}$ is the approach velocity of the aircraft given in Table 3 and $U_{\text {exit }}$ is the exit velocity of the water jet given in Table 2 . The experiment of Fritz and Hoffmann (2015) measured the size distribution $1.8 \mathrm{~m}$ downstream of the nozzle for all straight-stream nozzles (B. Fritz 2015, private communication). This value represents a correction from a value of $1.5 \mathrm{~m}$ reported in Fritz and Hoffmann (2015). Figure $1 \mathrm{~b}$ plots $\ell_{\text {relax }}$ as a function of drop radius for nozzle 1. Inspecting it together with the size distribution in Fig. 1a, we conclude that most of the droplets are following the airstream at the measurement station of the experiment. If this had not been the case and there had been a relative velocity large enough to give Weber numbers $\gtrsim 10$, then it would have been necessary to model further droplet breakup using a secondary breakup model (e.g., Apte et al. 2003).

\section{f. Flow field of two counterrotating wingtip vortices}

We consider an aircraft flying straight and level at altitude $z_{0}=0$ in aircraft coordinates. The velocity field of the airflow in the wake is denoted by lower case $\mathbf{u}(\mathbf{x})$. This velocity field consists of the free stream, $U_{\text {app }} \hat{\mathbf{x}}$ (where $U_{\text {app }}$ is the approach speed of the aircraft), superposed with the flow induced by a pair of counterrotating vortices with circulations $\pm \Gamma$. The centerline of each vortex is at spanwise location $y_{\text {vort }}= \pm b_{0}$. Because of their mutually induced velocity, the height $z_{\text {vort }}(x)$ of the vortex pair decreases with distance $x$ behind the wing as follows:

$$
z_{\text {vort }}(x)=z_{0}-W_{\text {desc }} t,
$$

(a) Drop size distribution of nozzle 1

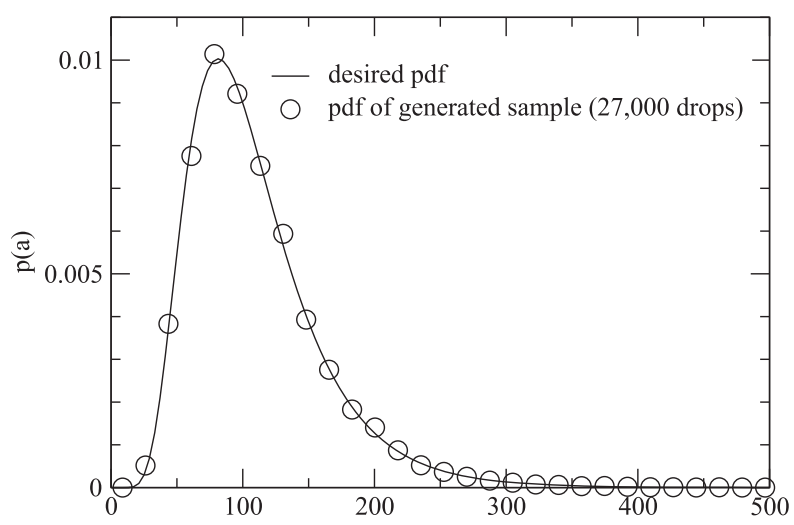

(b) Characteristic velocity relaxation distance

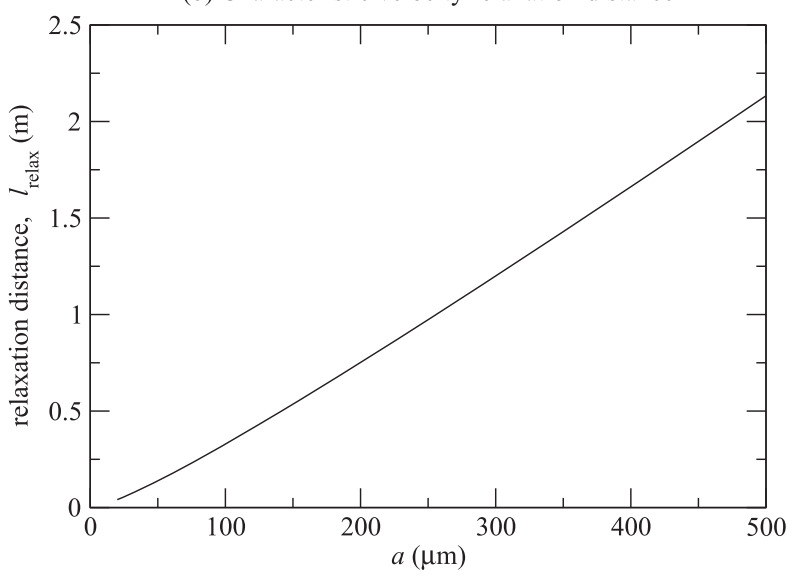

FIG. 1. (a) Probability density function (pdf) of the drop sizes produced by nozzle 1 with the conditions listed in Table 2 . The result is based on parameters provided by Fritz's Excel program. (b) Distance required for a droplet of a given size to begin moving with an imposed airflow.

where $W_{\text {desc }}=\Gamma / 2 \pi b_{0}$ is the descent speed of the vortex pair. The quantity $t=x / U_{\text {app }}$ is time, since the vortex at $x$ was shed from the wing.

Each vortex induces a circumferential velocity $u_{\theta}(r)$ in the cross plane $(y-z)$. For $u_{\theta}(r)$, a profile fit to flight data by Philippe Spalart (Boeing, 2015, private communication) is used,

$u_{\theta}=\frac{\Gamma}{2 \pi r} \begin{cases}1188.59 \eta^{2}, & \eta<0.0103 \\ {\left[1+(1.27+0.25 \log \eta)^{-14}\right]^{-1 / 14},} & \text { otherwise }\end{cases}$

where $\eta \equiv r / b_{0}$.

For an elliptically loaded wing, lifting line theory (Batchelor 1967) gives the vortex spacing as

$$
b_{0}=\frac{\pi}{4} b
$$


where $b$ is the wingspan, and the vortex circulation as

$$
\Gamma=\frac{W}{\rho_{a} U_{\text {app }} b_{0}},
$$

where $W$ is the aircraft weight. We use the parameters for a typical heavy aircraft given in Table 3.

\section{g. Calculation of the received signal and power}

A pulse-Doppler radar transmits a train of square wave pulses that modulate a carrier wave of frequency $f=2 \pi / \omega$. The duration of each pulse is $\tau$ and the pulse repetition frequency is $f_{\mathrm{PRF}}$. After each pulse is transmitted, the transmit-receive switch is set to the receive position and the incoming signal is sampled. Each sample at time $t$ is said to come from the range gate $r=c\left(t-t_{t}\right) / 2$, where $t_{t}$ is the transmit time of the pulse and the factor of 2 accounts for the round-trip. Throughout, we consider the case where the transmitting and receiving antennas are the same, the so-called monostatic case.

\section{1) Resolution SHELL}

An important concept is that of the resolution volume, $\mathscr{B}_{\tau}(t)$, at $t$, associated with a single pulse of finite duration, $\tau$ (e.g., Yuter 2003, p. 1836). It is defined as the volume from which a signal is received at a fixed $t$ due to scattering by the pulse. Let $t_{t}$ mark the beginning of the pulse at the transmitter. A signal from a scatterer at distance $r$ will be received in the time interval

$$
t-t_{t}=2 r / c+\xi \tau, \quad 0 \leq \xi \leq 1,
$$

where $\xi=0$ corresponds to the leading edge of the pulse and $\xi=1$ to its trailing edge. Solving (15) for $r$ gives

$$
r=c\left(t-t_{t}-\xi \tau\right) / 2, \quad 0 \leq \xi \leq 1 .
$$

Equation (16) defines a spherical shell (called the resolution volume) from which a signal is received at the fixed $t$. The next subsection describes how the complex voltage received at a given time is evaluated by summing the complex voltages from each droplet in the resolution volume.

\section{2) RECEIVED POWER AND SIGNAL-TO-NOISE RATIO}

The material in this subsection is adapted from the texts Doviak and Zrnić (1984) and Ishimaru (1978). Let the "voltage" of the transmitted pulse be (the real part of)

$$
V_{t}(t)= \begin{cases}V_{0} e^{i \omega t}, & t \leq \tau \\ 0, & \text { otherwise }\end{cases}
$$

where $V_{0}$ is a complex amplitude, $\tau$ is the pulse width, and voltage is defined such that the instantaneous transmitted power is $P_{t}(t)=V_{t}(t) V_{t}^{*}(t)$. The voltage at the input terminals of the receiver is (the real part of) the following summation over droplets:

$$
V(t)=\sum_{m=1}^{N_{d}} A_{m}(t) e^{\mathrm{i} \omega_{m}\left(t-2 r_{m} / c\right)},
$$

where $A_{m}$ is a complex scattering amplitude and

$$
\omega_{m} \equiv \omega\left(1-2 u_{m} / c\right)
$$

is the twice Doppler-shifted frequency ( $u_{m}$ being the radial velocity of the $m$ th droplet) and $r_{m}$ is the distance to each droplet. The summation in (18) is taken over the $N_{d}$ droplets in the resolution volume associated with the pulse.

The complex scattering amplitude due to each droplet is

$$
A_{m}=\left[\frac{\lambda^{2} \ell_{w} \ell_{B}}{(4 \pi)^{3}} \frac{G^{2}\left(\theta_{m}\right)}{r_{m}^{4}} \sigma_{b m}\right]^{1 / 2} V_{0} \exp \left(i \phi_{m}\right) .
$$

The first factor in (20), namely, [.] $]^{1 / 2}$, is copied from the square root of the radar equation (e.g., Doviak and Zrnić 1984, p. 34) for power, where $\lambda$ is the wavelength and $\sigma_{b m}$ is the backscattering cross section of each droplet. The function $G\left(\theta_{m}\right)$ is the gain function of the antenna at the droplet, which we have assumed to depend on its angle $\theta_{m}$ from the beam centerline. Two loss factors $(<1)$ have been included in $(20)$ : $\ell_{w}$ is the twoway waveguide loss and $\ell_{B}$ is the loss due to finite bandwidth of the receiver; values assumed for the present study are given in Table 4 . The last factor in (20), $\exp \left(i \phi_{m}\right)$, accounts for the phase shift induced by backscattering at the $m$ th droplet. The term $\sigma_{b m}$ and the phase shift $\phi_{m}$ will be discussed further in section $2 \mathrm{k}$.

Radar receivers have electronics that can obtain the real and imaginary parts (denoted $I$ and $Q$, respectively) of $V(t)$ and compute the instantaneous received power,

$$
P_{r}(t)=I(t)^{2}+Q(t)^{2}=V(t) V^{*}(t) .
$$

We note in passing that $I(t)$ and $Q(t)$ are the components of the real part of $V(t)$ that are in phase and $90^{\circ}$ out of phase with the transmitted carrier, respectively. Substituting (18) into (21) and splitting the sum into two parts, following Doviak and Zrnić (1984, section 4.1), gives

$$
P_{r}(t)=\sum_{m, n} A_{m} A_{n}^{*} \exp \left[i\left(\omega_{m}-\omega_{n}\right) t\right] \exp \left[-2 i\left(k_{m} r_{m}-k_{n} r_{n}\right)\right],
$$


TABLE 4. Radar parameters.

\begin{tabular}{|c|c|c|c|c|c|}
\hline Manufacturer & EEC & EEC & EEC & Metek & ProSensing \\
\hline Series & DWSR & DWSR & DWSR & & \\
\hline Model & $8501 \mathrm{~S}$ & $\mathrm{nnn} 1 \mathrm{C}^{\mathrm{a}}$ & $2001 X$ & MIRA-35 & W-SACR \\
\hline Frequency, $f(\mathrm{GHz})$ & 3 & 5.9 & 9.6 & 35.1 & 93.9 \\
\hline Peak power, $P_{t}(\mathrm{~kW})$ & 850 & $250-1000$ & 200 & 30 & 1.7 \\
\hline Reflector diameter (m) & 4.2 & 4.2 & 2.4 & $1.2,2.0$ & 0.9 \\
\hline Half-power beamwidth, $\theta_{b}$ & $1.83^{\circ}$ & $0.95^{\circ}$ & $0.95^{\circ}$ & $0.52^{\circ}, 0.31^{\circ}$ & $0.30^{\circ}$ \\
\hline Antenna gain, $G(\mathrm{~dB})$ & 39.5 & 45 & 45 & $50.4,53.5$ & 54.5 \\
\hline Pulse width, $\tau(\mu \mathrm{s})$ & $0.4-2$ & $0.2-3$ & $0.2-2$ & $0.1,0.2,0.4$ & $0.05-2$ \\
\hline Range resolution, $c \tau / 2(\mathrm{~m})$ & $60-300$ & $30-450$ & $30-300$ & $15,30,60$ & $7.5-300$ \\
\hline $\mathrm{PRF}(\mathrm{kHz})$ & $0.2-2.4$ & $0.2-2.4$ & $0.2-2.4$ & $2.5,5,10$ & $\leq 20$ \\
\hline$U_{\max }=c \mathrm{PRF} / 4 f\left(\mathrm{~m} \mathrm{~s}^{-1}\right)$ & $5-60$ & $2.5-31$ & $1.6-19$ & $5.3,11,21$ & $\leq 16$ \\
\hline Receiver noise figure (dB) & 2.0 & 2.0 & 2.0 & 6.2 & 6.0 \\
\hline Two-way waveguide loss ${ }^{\mathrm{b}}(\mathrm{dB})$ & 0.8 & 0.8 & 0.8 & 0.8 & 0.8 \\
\hline Finite bandwidth $\operatorname{loss}^{\mathrm{c}}(\mathrm{dB})$ & 1.8 & 1.8 & 1.8 & 1.8 & 1.8 \\
\hline Rain attenuation $^{\mathrm{d}}\left(\mathrm{dB} \mathrm{km}^{-1}\right)$ & 0.005 & 0.03 & 0.12 & 2 & 7 \\
\hline Minimum range $(\mathrm{m})$ & & & & $150^{\mathrm{e}}$ & \\
\hline
\end{tabular}

a The $\mathrm{nnn}$ is the power $(\mathrm{kW} ; 250,350,500$, or 1000$)$.

${ }^{\mathrm{b}}$ The only value provided to us was for MIRA-35. The value for the other radars was assumed to be the same.

${ }^{\mathrm{c}}$ The value for a perfectly matched filter has been assumed for all radars.

${ }^{\mathrm{d}}$ For a rainfall rate of $12.5 \mathrm{~mm} \mathrm{~h}^{-1}$ (medium to heavy rain).

${ }^{\mathrm{e}}$ MIRA-35 has full sensitivity beyond a range of $360 \mathrm{~m}$ (M. Bauer-Pfundstein 2015, private communication).

$$
\begin{aligned}
= & \sum_{m} A_{m} A_{m}^{*} \\
& +\sum_{m, n, m \neq n} A_{m} A_{n} \exp \left[i\left(\omega_{m}-\omega_{n}\right) t\right] \exp \left[-2 i\left(k_{m} r_{m}-k_{n} r_{n}\right)\right],
\end{aligned}
$$

where $k_{m} \equiv \omega_{m} / c$. Arguments for using only the first term in (23) in order to evaluate reflectivity - that is, for summing the powers received from each droplet-are given by Rayleigh (1945, p. 37), Beckmann (1962), and Doviak and Zrnić (1984, section 4.1). The important point, which was phrased eloquently by Rayleigh, is that it is not correct to say that the power in a single return from a random distribution of droplets is the sum of the powers scattered by each. Rather, the result is true only when a large ensemble of returns from a statistically stationary target is averaged. This is most easily seen when we consider the case when all the $A_{m}$ are equal (to unity, say). Then, the first term $T_{1}$ in (23) is $T_{1}=N_{d}$. If droplet distances are randomly distributed in the resolution shell (assumed to be several wavelengths wide), then the magnitude of the second term will be the average of the summands times the number of terms, that is, $T_{2} \approx N_{t}^{-1 / 2} \times N_{t}=N_{t}^{1 / 2} \approx N_{d}$, where $N_{t}=N_{d}\left(N_{d}-1\right)$ is the number of terms in the double sum. Hence, both terms in (23) are of similar magnitude. To make the second term smaller than the first, an ensemble average must be taken over many pulses. If the ensemble has $N_{s}$ phase-uncorrelated samples, then the second term will be $N_{s}^{1 / 2}$ smaller than the first.
In the present application, the wake descends through the beam and so the target is not stationary, strictly speaking. In section $3 \mathrm{~d}$ we will explicitly verify that, for our case, an average of (22) over a certain number of pulses does indeed yield the first term in (23). Note that a radar set does not have direct access to the first term in (23) —only we as simulators do.

To provide a measure of detectability, we will present the signal-to-noise ratio

$$
\mathrm{SNR} 1 \equiv P_{r 1} / P_{\text {noise }},
$$

where $P_{r 1}$ is the first term in (23) and the average noise power is

$$
P_{\text {noise }}=k_{B} T_{0} F_{N} / \tau,
$$

where $k_{B}=1.381 \times 10^{-23} \mathrm{~J} \mathrm{~K}^{-1}$ is Boltzmann's constant, $T_{0}=290 \mathrm{~K}$ is a reference temperature set by convention, and $F_{N}$ is the overall noise figure of the chain of components in the receiving cascade. Values for $F_{N}$ and $\tau$ are listed in Table 4 for each radar set.

Finally, since each computational droplet represents a multiplicity $M_{\text {actual }}$ of actual droplets, we have

$$
\sum_{m=1}^{N_{d}} \rightarrow M_{\text {actual }} \sum_{m=1}^{N_{\text {comp }}},
$$

where $N_{\text {comp }}$ is the number of computational droplets in the resolution volume. Note that all $M_{\text {actual }}$ copies of 
each computational droplet are assumed to be at the same location and therefore their scattered voltages at the receiver add constructively. This assumption does not bias SNR1, since its calculation involves summing individual scattered powers anyway. We claim that the statistics of individual pulse returns are also not affected by this assumption; this will be verified (section $3 \mathrm{e}$ ) in a computational test where the number of computational droplets is increased.

\section{h. Calculation of the Doppler spectrum}

Radars calculate a Doppler spectrum for a given spatial observation location by performing a fast Fourier transform (FFT) of complex voltage returns (at the same range gate) from a sequence of pulses separated by $\Delta t_{\text {pulses }}=1 / \mathrm{PRF}$, where PRF is the pulse repetition frequency. We shall do the same for the simulations. From a series of pulse returns, $V_{n}, n=0,1, \ldots, N_{\mathrm{FFT}}-1$, the normalized transform

$$
\hat{V}(k) \equiv \frac{1}{N_{\mathrm{FFT}}} \sum_{n=0}^{N_{\mathrm{FFT}}-1} V_{n} e^{-\mathrm{i} 2 \pi k n / N_{\mathrm{FFT}}}, \quad k=0, \ldots, N_{\mathrm{FFT}}-1,
$$

and then the power spectrum $S(k) \equiv \hat{V}(k) \hat{V}^{*}(k)$ is computed. Note that the frequency index $k$ corresponds to an actual frequency,

$$
k_{\text {actual }}= \begin{cases}k, & k \leq N_{\mathrm{FFT}} / 2 \\ k-N_{\mathrm{FFT}}, & N_{\mathrm{FFT}} / 2<k \leq N_{\mathrm{FFT}}-1 .\end{cases}
$$

The frequency $k_{\text {actual }}$ is in units of (the period of the sequence $)^{-1}=\left(N_{\mathrm{FFT}} \Delta t_{\mathrm{pulses}}\right)^{-1}$, so in units of $\mathrm{s}^{-1}$,

$$
f\left(k_{\text {actual }}\right)=\operatorname{PRF} k_{\text {actual }} / N_{\mathrm{FFT}} .
$$

Equating this to $-2\left(u_{\text {doppler }} / c\right) f$ gives the Doppler velocity associated with each $k_{\text {actual }}$. Finally, we state that we use the Hamming window (e.g., Harris 1978).

\section{i. Antenna gain function}

We assume a Gaussian beam with transmitted power flux (power per unit area) vector

$$
\mathbf{S}_{t}=A \exp \left(-\theta^{2} / \theta_{0}^{2}\right) \hat{\mathbf{r}},
$$

where $A$ is a coefficient, $\theta$ is the angle from the beam centerline, and

$$
\theta_{0}=(2 \sqrt{\ln 2})^{-1} \theta_{b}
$$

in terms of the half-power full-width $\theta_{b}$. The total power crossing a sphere of radius $r$ is

$$
P_{t}=4 \pi r^{2} \int_{0}^{\pi} \mathbf{S}_{t} \cdot \hat{\mathbf{r}} \sin \theta, \quad d \theta=2 \pi r^{2} \theta_{0}^{2} A
$$

for a narrow beam. Using the definition of the gain function, $G(\theta)$, we obtain

$$
G(\theta) \equiv \frac{4 \pi r^{2} S_{t}}{P_{t}}=\frac{2}{\theta_{0}^{2}} \exp \left(-\theta^{2} / \theta_{0}^{2}\right)
$$

\section{j. Radars included in the study}

Table 4 lists parameters of currently operational ground-based Doppler weather/cloud radars considered in the present study. The S-, C-, and X-band radars chosen are the Doppler Weather Surveillance Radar (DWSR) series manufactured by Enterprise Electronics Corporation (ECC; Enterprise, Alabama). Advanced Radar Corporation (ARC, Boulder, Colorado) makes quite similar $\mathrm{C}$ - and X-band radars, while Baron Services (Huntsville, Alabama) makes similar S-, C-, and X-band radars. The power and beamwidth values of the C-band Terminal Doppler Weather Radar (TDWR) deployed at many U.S. airports is subsumed by the range of values provided by the EEC C-band radar, and is therefore not included here.

A number of descriptions of Ka-band $(35 \mathrm{GHz})$ cloud radars have appeared in the literature (Hamazu et al. 2003; Görsdorf et al. 2015). In the present work, we use parameters of the MIRA-35 radar manufactured by Metek (Elmshorn, Germany), which is described in Görsdorf et al. (2015). This choice was motivated by its relatively high power $(30 \mathrm{~kW})$. Other Ka-band weather radars operational at the time of writing are (i) scanning 2-kW radars operated by the U.S. Department of Energy's Atmospheric Radiation Measurement (ARM) Climate Research Facility (Widener et al. 2012). (ii) The Copernicus 1-kW radar at Chilbolton Observatory (United Kingdom). (iii) An airborne 25-kW multifrequency (X, Ka, and $\mathrm{W}$ bands) radar developed by ProSensing that is being used by NASA's Langley Research Center for research into the detection and avoidance of supercooled water droplets.

The science and technology of W-band $(94 \mathrm{GHz})$ radars for cloud and precipitation research is reviewed in Kollias et al. (2007). For the present work we chose the W-band scanning ARM cloud radar (W-SACR), which was developed by the U.S. Department of Energy's ARM program (Widener et al. 2012; Kollias et al. 2014).

With increasing $f$, reflectivity increases as $f^{4}$ in Rayleigh's formula (ignoring Mie scattering corrections). Furthermore, the size of the antenna required to obtain the same beamwidth is reduced. The main drawback of high frequency is increased attenuation due to precipitation between the radar and target. For example, 
the last entry in Table 4 gives the attenuation rate in medium rain at $\mathrm{W}$ band as $7 \mathrm{~dB} \mathrm{~km}^{-1}$. A compensating factor is that when there is precipitation, the ambient humidity is also very high and so there is minimal evaporation, and if natural precipitation is present in the wake, it will also contribute to reflectivity.

\section{k. Mie cross section and phase shift}

Since we have rather large droplets in the present application and frequencies up to $94 \mathrm{GHz}$, the backscattering cross section and phase shift are obtained using Mie's formula instead of Rayleigh's approximation. We used the Bohren-Huffman Mie scattering (BHMIE) routine, available from Prof. Bruce T. Draine's website at Princeton University, and checked the results using the MIE vector code MIEV0 developed by Dr. Warren J. Wiscombe (NASA Goddard Space Flight Center).

Some understanding of notation is required to properly use these routines. Let the incident field be of unit magnitude and polarized in the 2-direction (defined to be perpendicular to the plane containing the incident and observer directions). For a spherical target, the scattered field in the far field is also polarized in the 2-direction and is given by

$$
E_{\mathrm{s} 2}(r, \theta)=\frac{e^{i k r}}{k r} f_{22}(\vartheta),
$$

where $f_{22}$ is complex, $k=2 \pi / \lambda, \vartheta$ is the angle of the observer relative to the direction of propagation of the incident wave, and $r$ is the distance from the center of the sphere. The backscattering cross section and phase shift are obtained as

$$
\begin{aligned}
\sigma_{b} & \equiv 4 \pi r^{2} \frac{\left|\mathbf{E}_{s}(\pi)\right|^{2}}{\left|\mathbf{E}_{i}\right|^{2}}=\frac{4 \pi}{k^{2}}\left|f_{22}(\pi)\right|^{2}, \\
\phi & =\arg \left[f_{22}(\pi)\right] .
\end{aligned}
$$

At the start of the reflectivity calculation at a given frequency, we tabulate the ratio $\sigma_{b} / \sigma_{b, \text { Rayleigh }}$ and the difference $\phi-\phi_{\text {Rayleigh }}$ as a function of droplet radius $a$. Rayleigh's formulas are (Ishimaru 1978, p. 19)

$$
\begin{aligned}
\sigma_{b, \text { Rayleigh }} & =4\left|K_{\varepsilon}\right|^{2}(k a)^{4}\left(\pi a^{2}\right), \\
\phi_{\text {Rayleigh }} & =\arg \left(K_{\varepsilon}\right),
\end{aligned}
$$

where $K_{\varepsilon}$ (a complex number) is given by

$$
K_{\varepsilon}=\frac{\varepsilon-1}{\varepsilon+2}
$$

The quantity $\varepsilon(f, T)$ is the complex dielectric constant of water; our convention of $e^{+\mathrm{i} \omega t}$ for the time dependence requires the imaginary part of $\varepsilon$ be positive for an absorbing material. It is a function of frequency and temperature, and was evaluated using the single Debye model of Liebe et al. (1991) as implemented in subroutines available from Prof. Chris O'Dell's website at Colorado State University. Since the droplet temperature is almost the same for all drops (the spread was $4^{\circ} \mathrm{C}$ at most), $\varepsilon$ is evaluated at the average temperature of all the drops in the trail.

Figure 2 displays $\sigma_{b}$ (normalized by the Rayleigh value) and $\phi$ as a function of droplet radius at the five frequencies considered in this work. At the largest radii in this work, $a \approx 600 \mu \mathrm{m}$, the error in using Rayleigh's cross section is about $20 \%$ at $35.1 \mathrm{GHz}$.

\section{l. Choice of ambient temperature and humidity}

Droplet evaporation calculations require specification of the ambient temperature and humidity. For guidance on appropriate choices, aviation routine weather reports (METARs) during 2000-14 were downloaded from online (http://mesonet.agron.iastate.edu/request/download. phtml) and processed for the five busiest airports in the United States. Figures $3 a$ and $3 b$ show the monthly averaged temperature and relative humidity $(\mathrm{RH})$, respectively, when IFR conditions prevailed. Figure $3 \mathrm{c}$ shows the percentage of reports that fall into the IFR category. One sees that the average RH is always above $90 \%$. To further synthesize these data, yearly averages were taken (Table 5). Among the five airports, Los Angeles International Airport (LAX), Los Angeles, California, has the highest rate of evaporation in IFR conditions on average, since it is the warmest and driest on average. Our choice is the IFR average for LAX, namely, $T=15.2^{\circ} \mathrm{C}$ and $\mathrm{RH}=92.7 \%$. Looking at the monthly data for the other four airports [Hartsfield-Jackson Atlanta International Airport (ATL), Georgia; Dallas-Fort Worth International Airport (DFW), Texas; Chicago O'Hare International Airport (ORD), Illinois; and John F. Kennedy International Airport (JFK), New York, New York], this appears to be a reasonable choice for them also: it is an approximate lower bound for their monthly $\mathrm{RH}$ and their temperature is higher only during the summer months, when IFR reports are low.

Since it is expensive for flight tests to wait for IFR conditions to occur, and it is desirable to have a wake sensor that can work in a wider variety of atmosphere conditions, we will also consider a case of lower humidity and higher temperature, namely, $\mathrm{RH}=60 \%$ and $T=20^{\circ} \mathrm{C}$.

\section{m. Radar placement with respect to the wake}

Here, we choose the radar location $\left(x_{\mathrm{rad}}, y_{\mathrm{rad}}, z_{\mathrm{rad}}\right)$ in aircraft-centered coordinates. Based on current wake 
(a) Backscattering cross-section, $\mathrm{T}=20 \mathrm{C}$, Draine routine

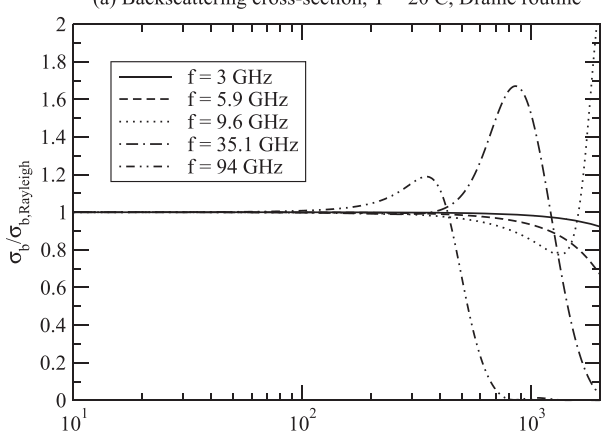

(c) Phase shift, $\mathrm{T}=20 \mathrm{C}$, Draine routine

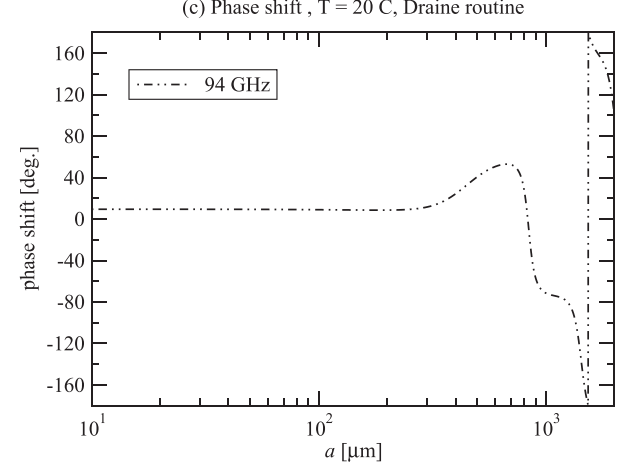

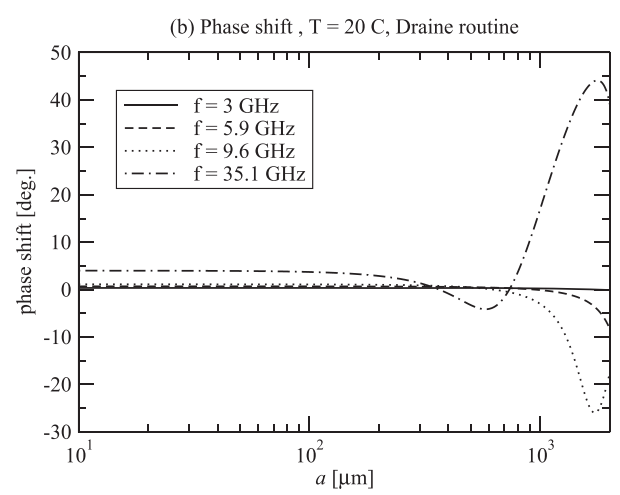

FIG. 2. Backscattering cross-section $\sigma_{b}$ (normalized by the Rayleigh value) and phase-shift $\phi$ vs droplet radius $a$ at the frequencies considered in this work. separations (Table 1), it should not be necessary to examine a wake more than $6 \mathrm{nmi}$ behind the aircraft. We therefore chose to present results for the reflectivity at $x=6 \mathrm{nmi}$, the worst case for droplet loss by evaporation and sedimentation. The radar is also placed on the ground at $x_{\text {rad }}=6 \mathrm{nmi}$ so it can view the $x=6 \mathrm{nmi}$ wake cross section at normal incidence. Next, we assume that the aircraft is at the touchdown point. At $6 \mathrm{nmi}$ from the touchdown point, the altitude of an aircraft flying a $3^{\circ}$ glide slope is $H=582 \mathrm{~m}$. Therefore, the vertical coordinate of the radar is $z_{\mathrm{rad}}=-582 \mathrm{~m}$. For purposes of this study, we assume an aircraft flying straight and level at this altitude. Initial flight tests would also presumably have the aircraft fly straight and level. In this case, since the vortex pair descends at a speed of $W_{\text {desc }}=1.75 \mathrm{~m} \mathrm{~s}^{-1}$, its axis makes a downward angle of $1.7^{\circ}$ relative to the horizontal. For an aircraft on a $3^{\circ}$ glide slope, the vortex axis would therefore be $1.3^{\circ}$ upward from the wing. This difference in the angle of the wake axis is expected to have a very small effect on reflectivity.

To place the radar laterally with respect to the wake, we imagine several parallel approaches that are monitored by the same radar. The largest separation between parallel runways is about $5000 \mathrm{ft}$ (Doyle and McGee 1998). At $6 \mathrm{n}$ mi from touchdown, the lateral width of the Instrument Landing System (ILS) approach is $3182 \mathrm{ft}$ for a standard $5^{\circ}$ splay, and we imagine an aircraft that has strayed to the outer edge of this zone. If the radar is placed in the middle of the two farthest runways, we obtain a lateral distance of $0.67 \mathrm{n} \mathrm{mi}$. In the presence of a crosswind, we imagine that the wake would be monitored for as long as it remained between the outer edges of the left and right ILS zones. In conclusion, we select $\left(x_{\text {rad }}, y_{\text {rad }}, z_{\text {rad }}\right)=(6 \mathrm{n} \mathrm{mi},-0.67 \mathrm{n} \mathrm{mi},-582 \mathrm{~m})$ relative to the aircraft.

The elevation angle of the radar beam from this location varies between $10.6^{\circ}$ and $17.1^{\circ}$ as the scanned range of $z$ on the wake center plane varies between $z=-350$ and $-200 \mathrm{~m}$ (see Fig. 4). Since the beamwidth of the radar likely to be used is $\leq 1^{\circ}$, ground reflection will be small. To significantly reduce ground and structure clutter, the radar can be placed directly under the flight path. This would require a separate radar for each parallel runway. Another issue is the loss of radar sensitivity at smaller ranges; for MIRA-35 this happens for $r<360 \mathrm{~m}$ (M. Bauer-Pfundstein 2015, private communication). However, this loss is probably offset by the increase in power from the $r^{4}$ factor in (20).

\section{Results}

\section{a. Signal-to-noise ratio for the IFR case}

We begin by considering IFR ambient conditions $\left(\mathrm{RH}=92.7 \%, T=15.2^{\circ} \mathrm{C}\right)$ chosen as described in section 21. Nozzle 1 from Table 2 is used and the parameters for 
(a) Temperature

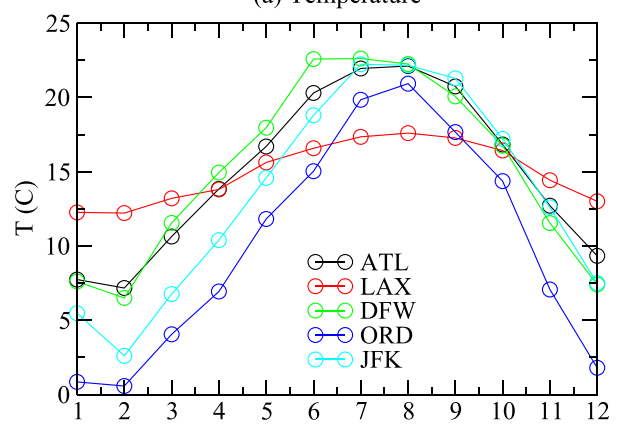

(c) Percent IFR reports

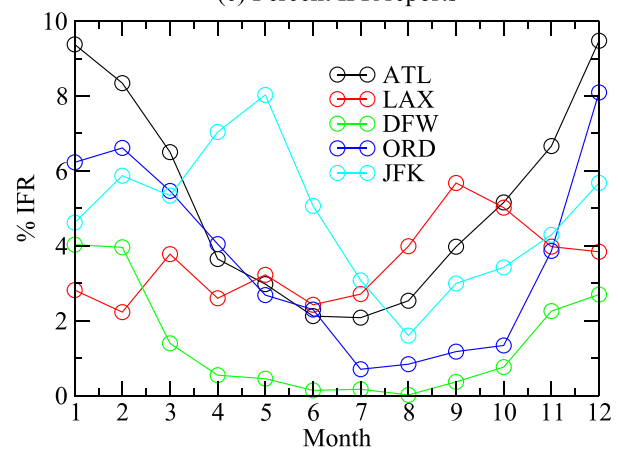

the injected square of droplets, described in section 2e, are $n_{\text {square }}=15, n_{x}=120$, and $w_{\text {square }}=1 \mathrm{~m}$. Figure 4 shows simulated values of SNR1, calculated using (24), for the five radars listed is Table 4. Each SNR1 plot is an instantaneous range-elevation scan of the $x=6 \mathrm{nmi}$ cross section of the wake, and each location on the plot corresponds to the midradius of a resolution shell along the beam centerline. The pulse width is chosen to be $\tau=0.2 \mu$ s for all the radars except for DWSR-8501S, in which case the lowest available $\tau$ of $0.4 \mu$ s is used. Droplets in a 30-m-thick axial slab centered at $x=6 \mathrm{n} \mathrm{mi}$ are shown in Fig. 4a. Because of centrifugation, larger droplets lie at greater distances from the vortex center, which is devoid of droplets. The largest droplets fall out of the wake due to gravity after being centrifuged. Except for the S-band radar, all radars give SNR1 $>10 \mathrm{~dB}$ at most points surrounding the vortices; the reflectivity is higher for the higher-frequency radars. The W-SACR gives the highest reflectivity despite having the smallest pulse power.

For all radars, there is a drop in reflectivity near the 2 and 4 o'clock positions for the left vortex ( 8 and 10 o'clock positions for the right vortex). This manifests as a crescent wrench-shaped reflectivity pattern that is most prominent for the DWSR 2001X radar. A plot of droplet number density indicated a drop at these locations. How this is related to the vortex flow field remains to be elucidated. (b) Relative Humidity

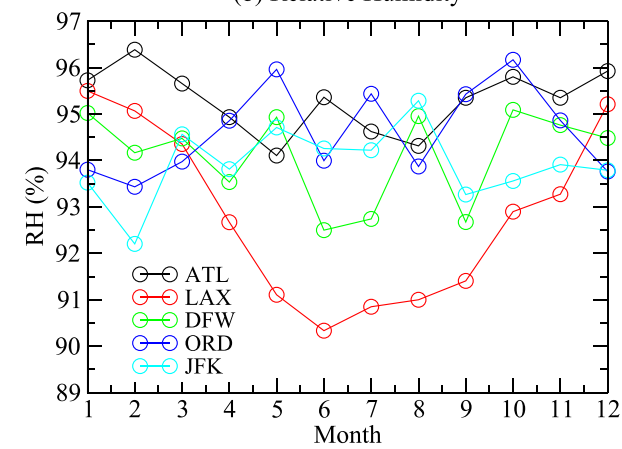

FIG. 3. Temperature and humidity (averaged by month) when IFR conditions prevail at the five busiest U.S. airports.

\section{b. Effect of pulse width and beamwidth}

Consider a distribution of droplets that is homogeneous in the range of pulse volumes (resolution length $X$ beam section) considered. Then, from (20) the dependence of received power $\left(P_{r}\right)$ on pulse width $(\tau)$ and beamwidth $\left(\theta_{b}\right)$ is

$$
P_{r} \propto\left(R \theta_{b}\right)^{2}(c \tau / 2) G^{2} \propto \tau \theta_{b}^{-2},
$$

since the antenna gain $G \propto \theta_{b}^{-2}$. Figure 5a shows the effect of using the 4-times-smaller pulse width of $\tau=0.05 \mu \mathrm{s}$ available in the W-SACR; compare this with Fig. 4f. One sees that some of the spiral structure of the droplet pattern is now resolved at the expense of a 6-dB drop in SNR1; this is in accordance with (40). Figure $5 \mathrm{~b}$ shows the difference in SNR1 $(\mathrm{dB})$ when the $\theta_{b}=0.31^{\circ}$ beam is used for the MIRA-35 radar instead of the $\theta_{b}=0.52^{\circ}$ beam. The difference agrees approximately with the difference of $4.5 \mathrm{~dB}$

TABLE 5. Yearly averaged temperature and humidity when IFR conditions prevail at the five busiest U.S. airports.

\begin{tabular}{lccc}
\hline \hline Airport & $T\left({ }^{\circ} \mathrm{C}\right)$ & $\mathrm{RH}(\%)$ & IFR reports $(\%)$ \\
\hline ATL & 12.5 & 95.5 & 5.3 \\
LAX & 15.2 & 92.7 & 3.5 \\
DFW & 9.7 & 94.5 & 1.4 \\
ORD & 5.3 & 94.2 & 3.7 \\
JFK & 12.1 & 93.9 & 4.8 \\
\hline
\end{tabular}


(a) Droplets in a 30 meter slab colored by size in microns

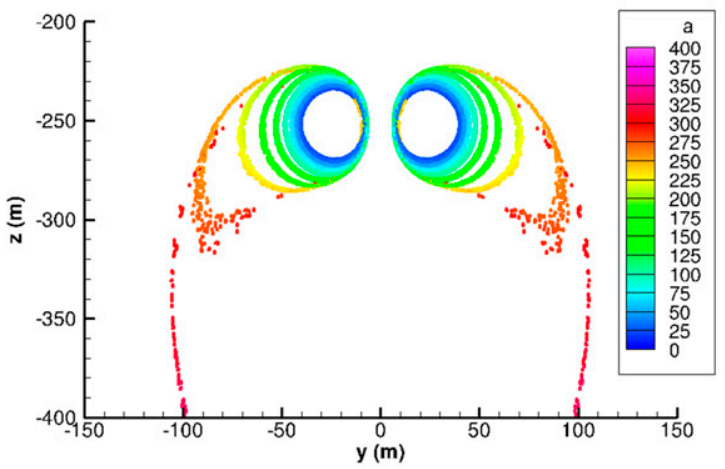

(c) DWSR 10001C, $\tau=0.2 \mu \mathrm{s}, \theta_{\mathrm{b}}=0.95 \mathrm{deg}$.

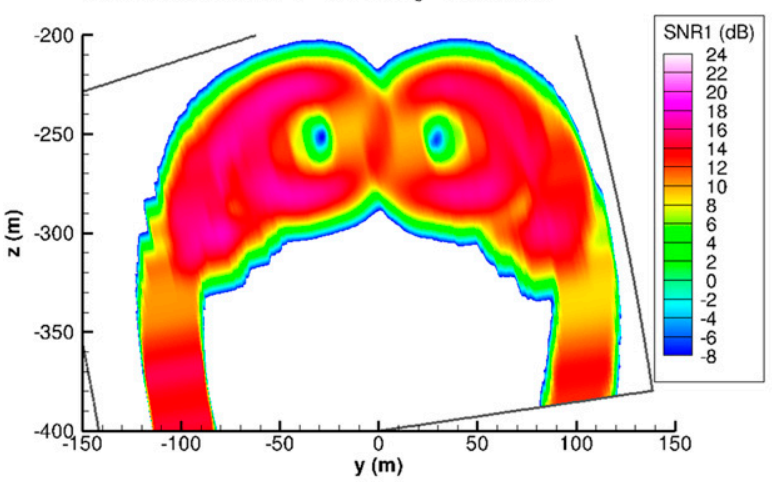

(e) Mira-35, $\tau=0.2 \mu \mathrm{s}, \theta_{\mathrm{b}}=0.52 \mathrm{deg}$.

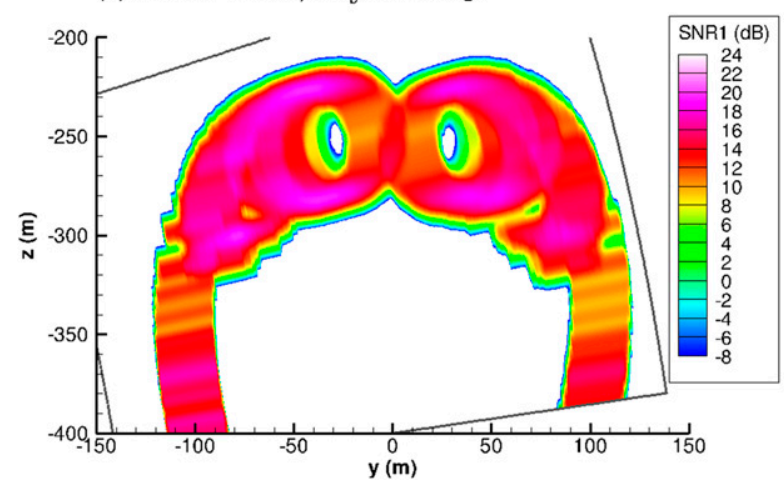

(b) DWSR $8501 \mathrm{~S}, \tau=0.4 \mu \mathrm{s}, \theta_{\mathrm{b}}=1.83 \mathrm{deg}$.

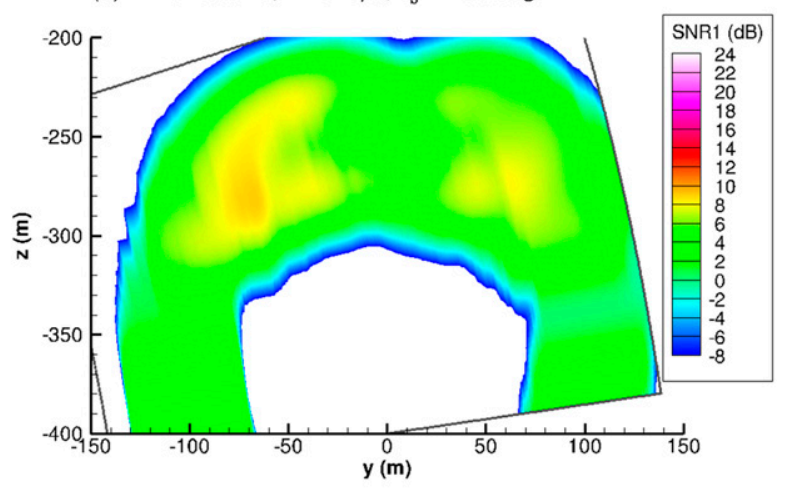

(d) DWSR 2001X, $\tau=0.2 \mu \mathrm{s}, \theta_{\mathrm{b}}=0.95 \mathrm{deg}$.

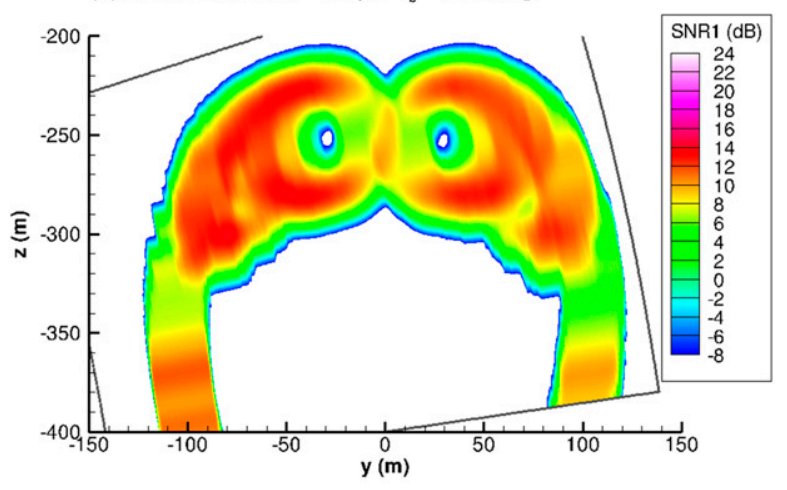

(f) $W$-SACR, $\tau=0.2 \mu \mathrm{s}, \theta_{b}=0.30 \mathrm{deg}$.

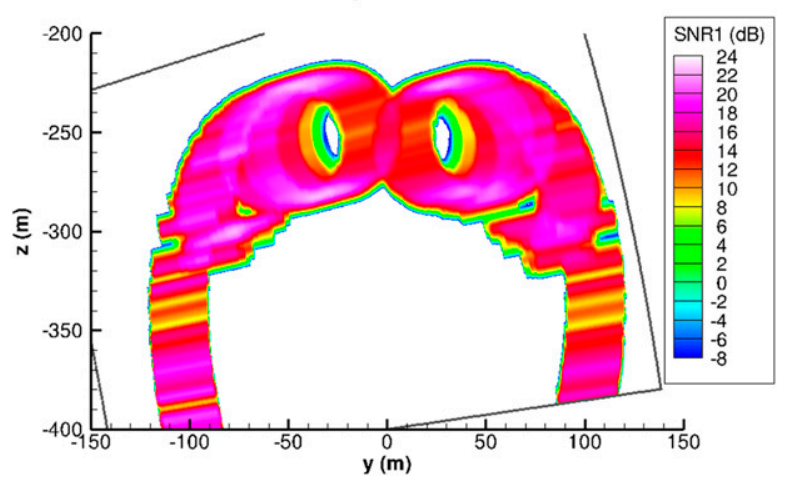

FIG. 4. Simulated SNR1 for five radars in a range-elevation scan of the $x=6 \mathrm{n}$ mi cross section behind the aircraft. (a) Droplets colored by radius $(\mu \mathrm{m})$. IFR ambient conditions: $\mathrm{RH}=92.7 \%, T=15.2^{\circ} \mathrm{C}$. Nozzle 1 .

given by (40). Locations where the difference is different from this are those where the droplet distribution is not homogeneous.

\section{c. Insensitivity to initial condition}

To test the sensitivity to initial conditions, instead of injecting droplets in a regular grid pattern on each square, droplets were randomly placed in the squares. Figures $6 \mathrm{a}$ and $6 \mathrm{~b}$ show that both the droplet configuration at $x=6 \mathrm{nmi}$ and SNR1 for DWSR-2001X are changed very little; compare with Figs. 4a and 4d, respectively. We expect this to be true for all the radars as well. In another test (Figs. $6 \mathrm{c}$ and $6 \mathrm{~d}$ ), $w_{\text {square }}$ was reduced from $1 \mathrm{~m}$ to $50 \mathrm{~cm}$, keeping the number of droplets fixed. This increases the initial number density in $y z$ and, to keep the spacing the same in the streamwise $(x)$ direction, the injection interval $\Delta t_{\text {inject }}$ was also halved. One might think that this would increase the number density downstream. However, the flow tends both to reduce number density (where there is rotation) and increase it (where there is strain), and 
(a) W-SACR, $\tau=0.05 \mu \mathrm{s}, \theta_{\mathrm{b}}=0.30 \mathrm{deg}$.

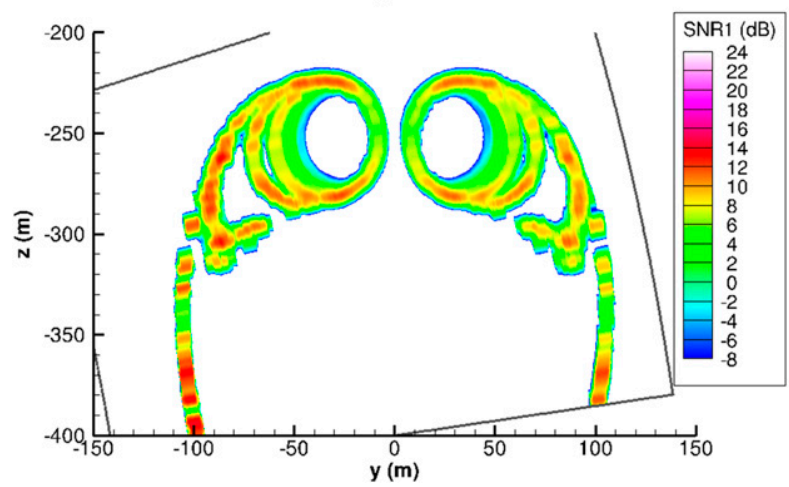

(b) SNR1 (dB) difference, $\theta_{\mathrm{b}}=0.31$ deg. vs. $0.52 \mathrm{deg}$. Mira-35, $\tau=0.2 \mu \mathrm{s}$.

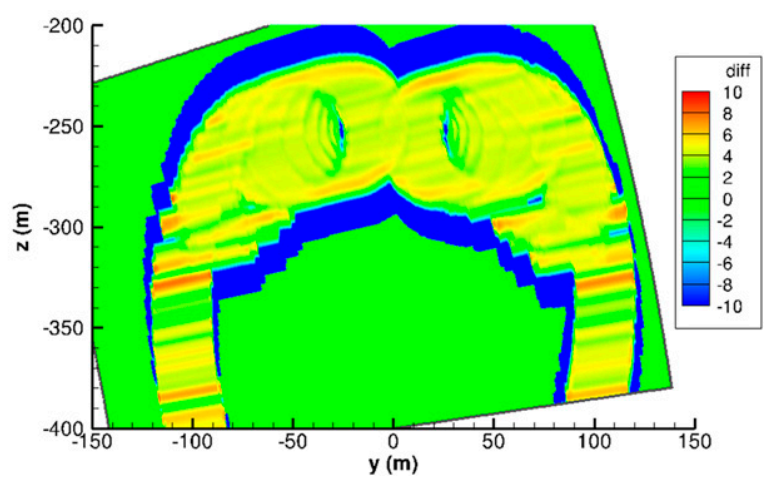

FIG. 5. Effect of pulse width and beamwidth on simulated SNR1. (a) W-SACR with a pulse width of $0.05 \mu$ s. (b) Difference in SNR1 (dB) when MIRA-35 is used with a beamwidth of $\theta_{b}=0.31^{\circ}$ compared to $\theta_{b}=0.52^{\circ} ; x=6 \mathrm{n}$ mi cross section of the wake, and IFR ambient conditions. Nozzle 1.

eventually, the number density tends to a distribution that is mostly independent of the initial condition.

\section{d. Pulse-to-pulse fluctuation and averaging}

The SNR1 results in section 3a were based on using the first term in (23), which sums the powers reflected by

(a) Droplets in a 30 meter slab colored by size in microns. Initial condition: random on $100 \mathrm{~cm}$ by $100 \mathrm{~cm}$ squares.

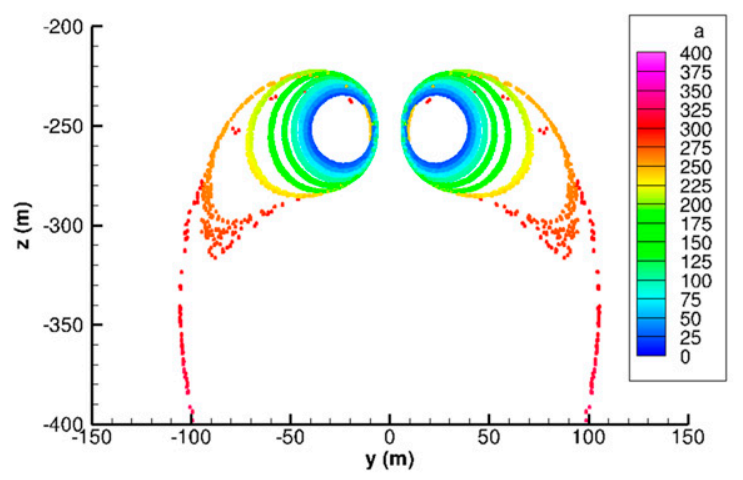

(c) Droplets in a 30 meter slab colored by size in microns. Initial conditon: regular grid on $50 \mathrm{~cm}$ by $50 \mathrm{~cm}$ squares.

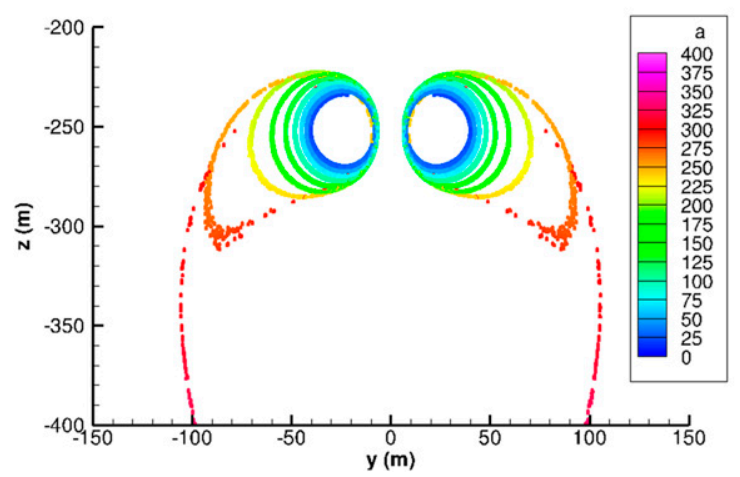

individual droplets. It was argued that for a statistically stationary target, this should equal the average power from many pulses. In the present case, the droplet configuration is not spatially homogeneous and is descending through a fixed beam. Hence, the question arises whether the powers returned from a sequence of (b) DWSR 2001X, $\tau=0.2 \mu \mathrm{s}, \theta_{b}=0.95 \mathrm{deg}$. Initial condition: random on $100 \mathrm{~cm}$ by $100 \mathrm{~cm}$ squares.

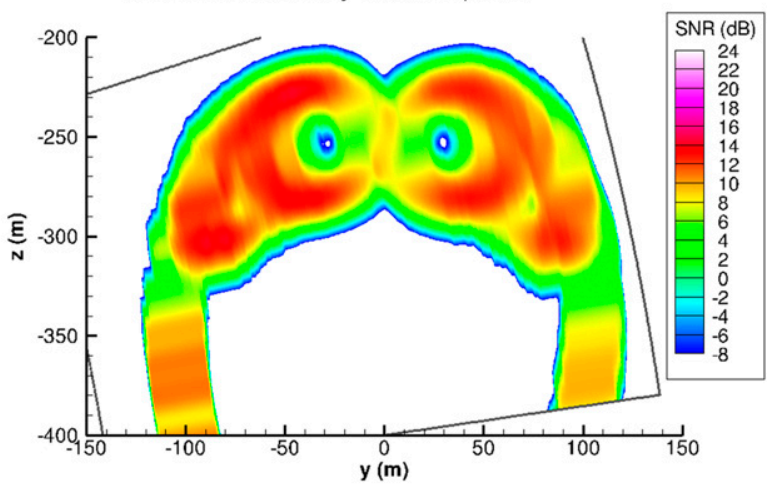

(d) DWSR 2001X, $\tau=0.2 \mu \mathrm{s}, \theta_{b}=0.95 \mathrm{deg}$. Initial condition: regular grid on $50 \mathrm{~cm}$ by $50 \mathrm{~cm}$ squares.

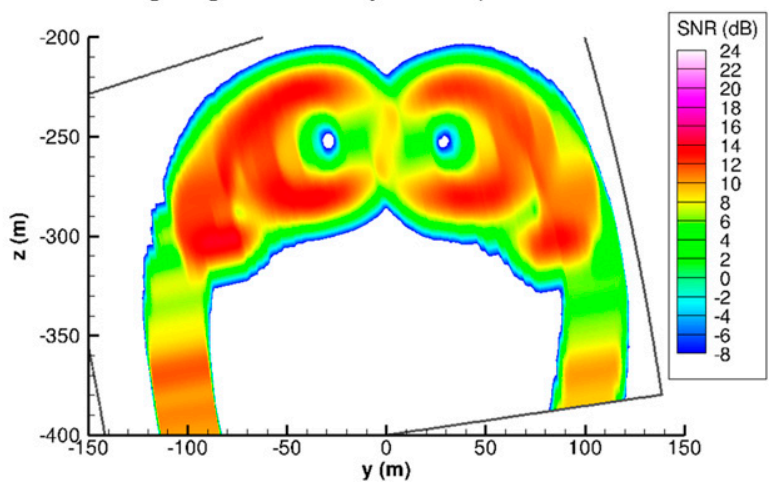

FIG. 6. Insensitivity to the initial condition. (a),(b) Droplets were placed randomly on each $1 \mathrm{~m} \times 1 \mathrm{~m}$ square. (c),(d) Droplets were arranged on a regular grid on each $50 \mathrm{~cm} \times 50 \mathrm{~cm}$ square. 
(a) DWSR 2001X, PRF $=2 \mathrm{kHz}, \tau=0.2 \mu \mathrm{s}, \theta_{\mathrm{b}}=0.95 \mathrm{deg}$.

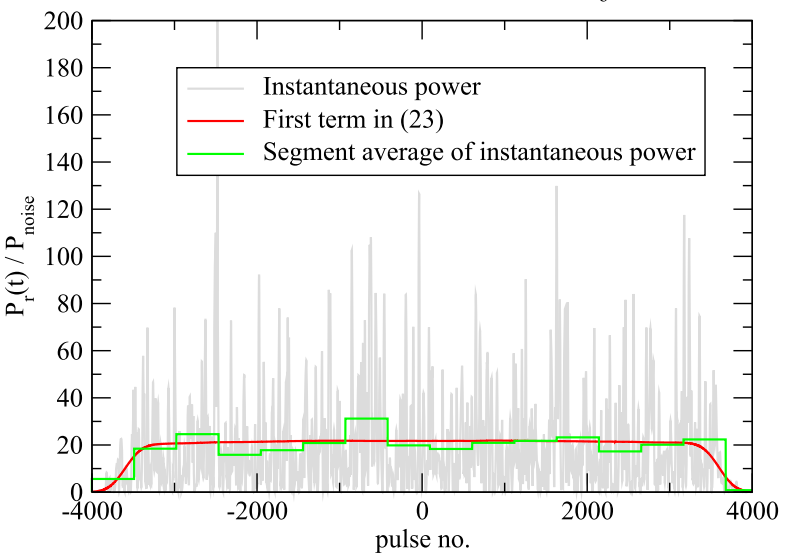

(c) W-SACR, PRF $=20 \mathrm{kHz}, \tau=0.2 \mu \mathrm{s}, \theta_{\mathrm{b}}=0.30 \mathrm{deg}$.

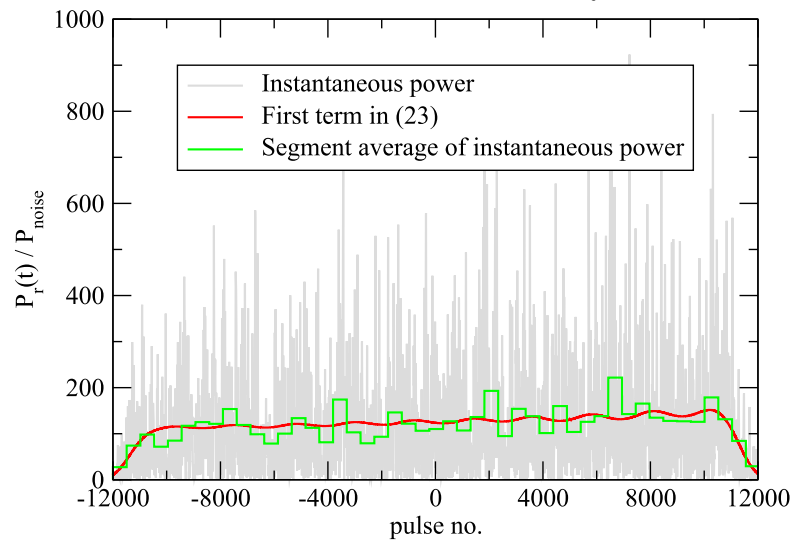

pulses can be considered to be statistically stationary in a certain interval, and if so, how many pulses are sufficient to recover the SNR1 values presented.

To obtain complex voltage returns from a sequence of pulses, one needs to evolve the wake in time; however, the method that was described in section 2 gives a trail of droplets at a single instant of $t$. To evolve this configuration to time $t+\Delta t$, the configuration at $t$ is translated horizontally by $\Delta x=-U_{\text {app }} \Delta t$; that is, the droplet trail is assumed to be invariant in a reference frame moving to the left with the airplane. This procedure does not correspond exactly to reality, but it captures both the rotation of droplets around the vortices and their vertical descent with time at a fixed location. The received complex voltage is evaluated using (18) at a sequence of times separated by the pulse repetition period, keeping the resolution volume centered at $(x, y, z)=$ $(6 \mathrm{~nm},-50 \mathrm{~m},-230 \mathrm{~m})$. This location corresponds to the upper SNR1 peak of the crescent wrench in Fig. 4. The value of the PRF was chosen to be at or close to the highest value available for each radar.

Figure 7 shows the result for a period during which a cluster of droplets enters and leaves the beam. To save (b) MIRA-35, PRF $=10 \mathrm{kHz}, \tau=0.2 \mu \mathrm{s}, \theta_{\mathrm{b}}=0.52 \mathrm{deg}$.

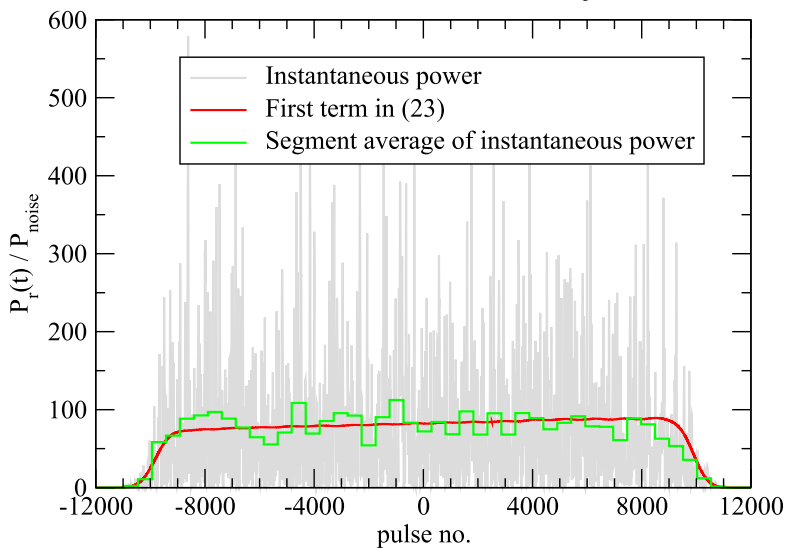

FIG. 7. Instantaneous power $P_{\mathrm{r}}(t)$ received from the same range gate due to a sequence of transmitted pulses (gray line). The range cell is at $(x, y, z)=(6 \mathrm{nmi},-50 \mathrm{~m},-230 \mathrm{~m})$. Received powers averaged over segments 512 pulses long (green line). First term in (23) (red line). (a)-(c) Same cases as in Fig. 4d-f, respectively.

space, plots for the S- and C-band radars have been omitted; they tell the same story. The power in individual pulse returns is shown in gray. The total number of active pulses changes from radar to radar because their beam widths and PRFs are different. In particular, the period of activity was found to equal the time it would take the vortex pair to descend through roughly one-third of the vertical projection of the half-power beamwidth. The average of pulse powers is shown in green over an averaging segment whose length is 512 pulses. The red curve shows the value of SNR1. Our assumption was that SNR1 (red) should equal the green level. This is seen to be true to a good degree. The fluctuations are due to statistical error and were found to decrease with increasing the averaging interval. It is worth remembering here that the radar has access to only the individual pulse returns and their average (e.g, the green values); only the simulation has access to the red curve (SNR1).

\section{e. Convergence of pulse statistics}

Recall that each computational droplet at a single location represents $M_{\text {actual }}$ actual droplets located at different positions; in fact, $M_{\text {actual }} \approx 100$ in the calculations 
presented. It was claimed (section $2 \mathrm{~g}$ ) that this should not affect pulse statistics, provided the number of computational droplets is sufficiently large. To verify this, the number of computational droplets was increased by four. Random placement of droplets was employed in the injected squares. Four realizations of the droplet trail were generated using different random number seeds for the initial size distribution and droplet placement. The four realizations were then merged into one trail for the radar reflectivity calculation. Figure 8 shows that the probability density $p(|V|)$ of the modulus $|V|$ of complex voltage is unchanged by the resolution refinement. The probability densities are very well fit by the Rayleigh distribution (Beckmann 1962),

$$
p(|V|)=\frac{|V|}{\sigma_{\mathrm{R}}^{2}} \exp \left(-|V|^{2} / 2 \sigma_{\mathrm{R}}^{2}\right)
$$

having the same mean as the data. The Rayleigh distribution results when the scattering amplitude is the same for all droplets and the phases are uniformly distributed. The case selected is the same as that shown in Fig. 7b (apart from the random placement of droplets in the injected squares). Pulses in the interval of stationarity were chosen, namely, pulse number $\in[-8000,8000]$.

\section{f. A non-IFR condition}

It would be valuable to have the capability to detect wakes in non-IFR conditions. Furthermore, in a flight test study of the feasibility of the present proposal, it would be too costly to wait until IFR conditions occur before a test can be conducted. For this reason it is of interest to know what reflectivity is obtained at less humid and less cold conditions. We chose $\mathrm{RH}=60 \%$ and $T=20^{\circ} \mathrm{C}$.

With the previous choice of $n_{\text {square }}=15$ and $n_{x}=120$ as injection parameters, it was found that a high rate of evaporation resulted in a small number of computational droplets remaining near $x=6 \mathrm{~nm}$. This increased statistical error. To reduce sampling error, an ensemble of 10 trails was computed with different random number seeds for the droplet size sample. The ensemble was then combined into a single trail for the reflectivity analysis. As a result, the total number of computational droplets is so large that each one presents only 9.8 true droplets in the reflectivity analysis.

Figure 9 shows that only the high-frequency radars, MIRA-35 and W-SACR, give positive values of SNR1 $(\mathrm{dB})$ in the vicinity of the vortices, and even these values are marginal. To increase SNR1, the number of nozzles could be increased; for instance, four nozzles on each side of the aircraft would increase SNR1 by $6 \mathrm{~dB}$.

There is a powerful method that enables detection even when SNR1 $(\mathrm{dB})<0$. It comes at the cost of increased dwell and processing time. We learned about the method from notes on the sensitivity of the MIRA35 radar given to us by Matthias Bauer-Pfundstein (Metek). It is also briefly described in Görsdorf et al. (2015, p 680). The idea is that in a discrete Fourier transform, the noise is spread equally to all the frequency bins, whereas the spectrum of the signal is confined to only a few of the bins (the latter is true provided the probability distribution of droplet velocities in the resolution cell is narrow compared to $2 U_{\max }$ for the radar; for MIRA-35, e.g., at PRF $=10 \mathrm{kHz}$, we have $2 U_{\max }=42 \mathrm{~m} \mathrm{~s}^{-1}$ and so this is unlikely to be an issue.) Hence, an FFT effectively reduces the noise by a factor of $N_{\text {FFT }}$.

To investigate this technique, complex (Gaussian distributed) white noise with a mean power equal to $P_{\text {noise }}$ for the radar was added to complex voltages of pulse returns. Illustrative results are shown in Fig. 10 for $N_{\mathrm{FFT}}=512$. Figures $10 \mathrm{a}$ and $10 \mathrm{~b}$ are for a range cell centered at the left white dot in Fig. 9e, where $\mathrm{SNR} 1=-2.7 \mathrm{~dB}$. Figures $10 \mathrm{c}$ and $10 \mathrm{~d}$ are for the right white dot, where SNR1 is even lower, namely, $-7.2 \mathrm{~dB}$. Consider Figs. 10a and 10b: An averaging of pulse power returns by the radar would give values (the green line) only slightly above the noise, not enough for a positive detection. Averaging the Doppler spectra from $10 \mathrm{seg}$ ments gives Fig. $10 \mathrm{~b}$ with a peak $15 \mathrm{~dB}$ above the noise. In the present example, this would require a dwell time of $0.5 \mathrm{~s}$ for each elevation angle. For the second location, where SNR1 is weaker, the Doppler spectrum has a peak that is about $10 \mathrm{~dB}$ above the noise (using the same dwell time). The contribution to the Doppler spectra from noise can be estimated as $P_{\text {noise }} \sigma_{\text {win }}^{2} / N_{\mathrm{FFT}}=$ $-162-\mathrm{dB} \mathrm{W}$, where $P_{\text {noise }}=8.34 \times 10^{-14} \mathrm{~W}$ is the average

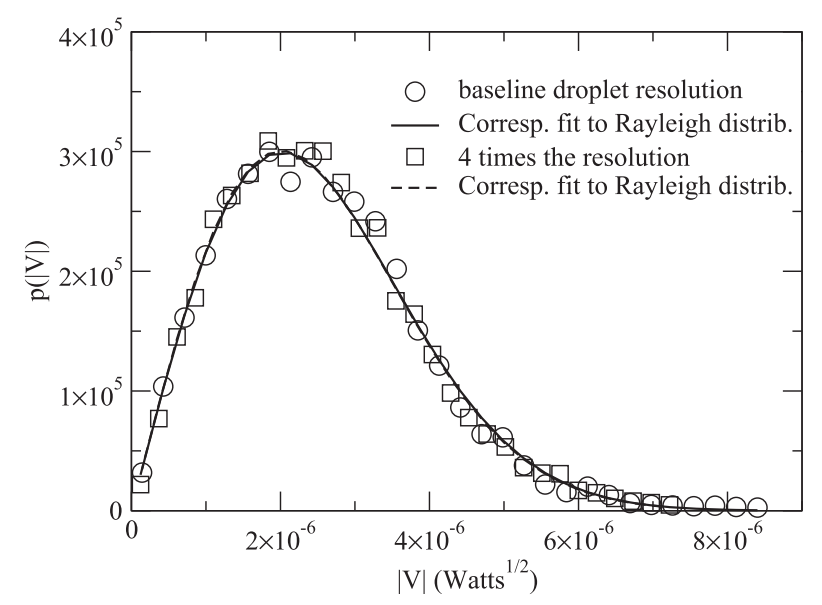

FIG. 8. Convergence of pulse statistics when the number of computational droplets is increased by four. The same case as in Fig. $7 \mathrm{~b}$ is used (MIRA-35 radar). Note: The solid and dashed lines are nearly coincident. 
(a) Droplets in a 30 meter slab colored by radius in microns.

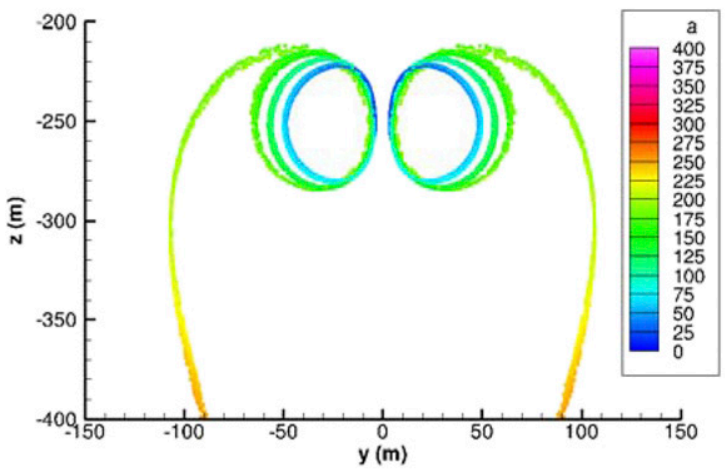

(c) DWSR 10001C, $\tau=0.2 \mu \mathrm{s}, \theta_{\mathrm{b}}=0.95 \mathrm{deg}$

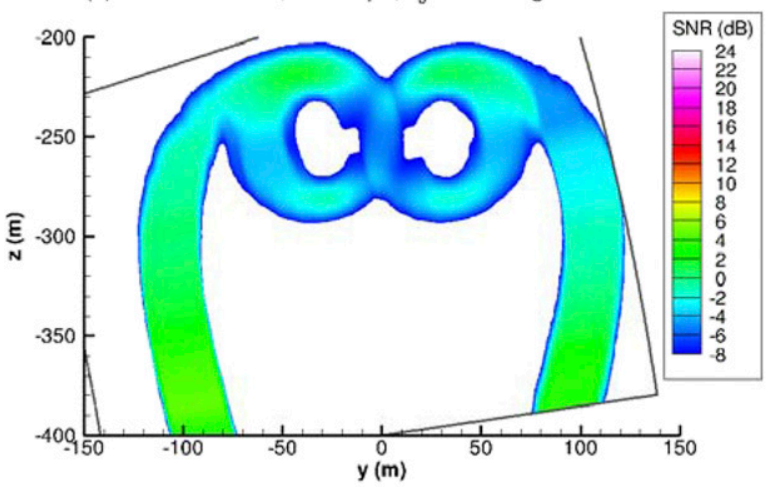

(e) MIRA-35, $\tau=0.2 \mu \mathrm{s}, \theta_{\mathrm{b}}=0.52 \mathrm{deg}$.

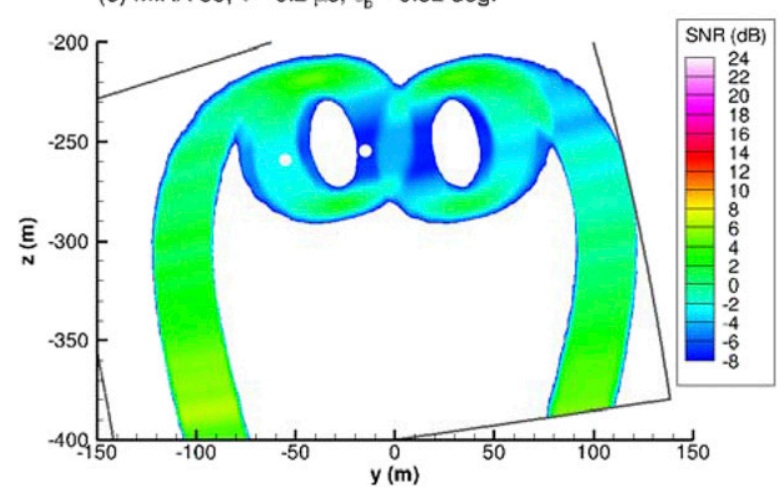

(b) DWSR 8501S, $=0.4 \mathrm{~s}, \theta_{\mathrm{b}}=1.83 \mathrm{deg}$.

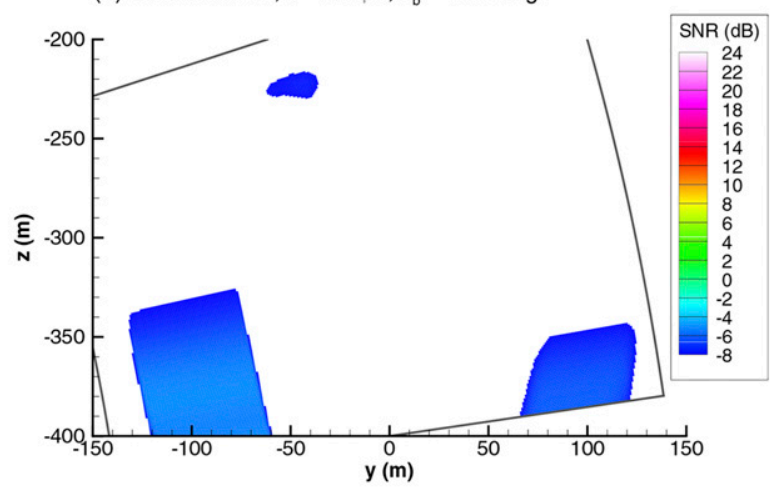

(d) DWSR 2001X, $\tau=0.2 \mu \mathrm{s}, \theta_{\mathrm{b}}=0.95 \mathrm{deg}$.

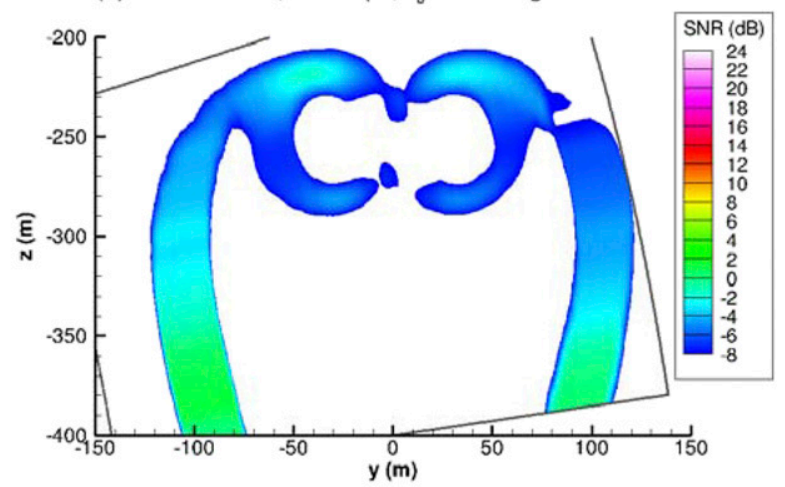

(f) W-SACR, $\tau=0.2 \mu \mathrm{s}, \theta_{b}=0.30 \mathrm{deg}$.

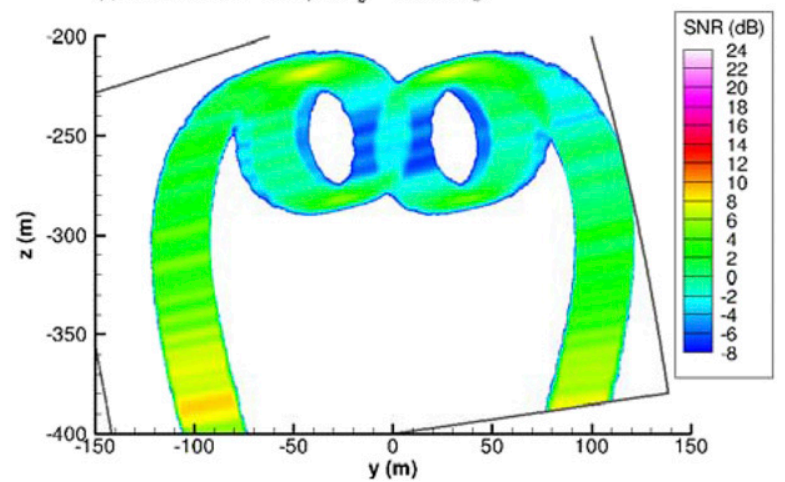

FIG. 9. SNR1 for a non-IFR condition $\left(\mathrm{RH}=60 \%, T=20^{\circ} \mathrm{C}\right)$. A range-elevation scan of the wake cross section $6 \mathrm{~nm}$ behind the aircraft is shown. White circles in (e) are points for which a spectral analysis is presented in Fig. 10.

noise power for the MIRA-35 radar and $\sigma_{\text {win }}^{2}=0.397$ is the mean square of the Hamming window.

\section{g. Power-weighted average radial velocity}

It has been stated (Doviak and Zrnić 1984, section 5.2) that the first moment of the Doppler spectrum is the radial velocity of droplets in the resolution volume, weighted by their individual scattered powers. This is reasonable, although we have neither encountered nor attempted a proof of it starting from
(22). Figure 11 displays the power-weighted average radial velocity corresponding to the cases previously shown in Fig. 4. Only points where SNR $(\mathrm{dB})>0$ are shown. The actual radial velocity (with respect to the radar) of the gas is shown in Fig. 11a. The radar data appear as a filtered version of the actual velocity and, due to particle centrifugation, are unable to detect the maximum value of $19.8 \mathrm{~m} \mathrm{~s}^{-1}$ in the vortex core. Nevertheless, the radars give a good representation of the gas velocity where particles are present. To 
(a) Received pulse powers of signal + white noise

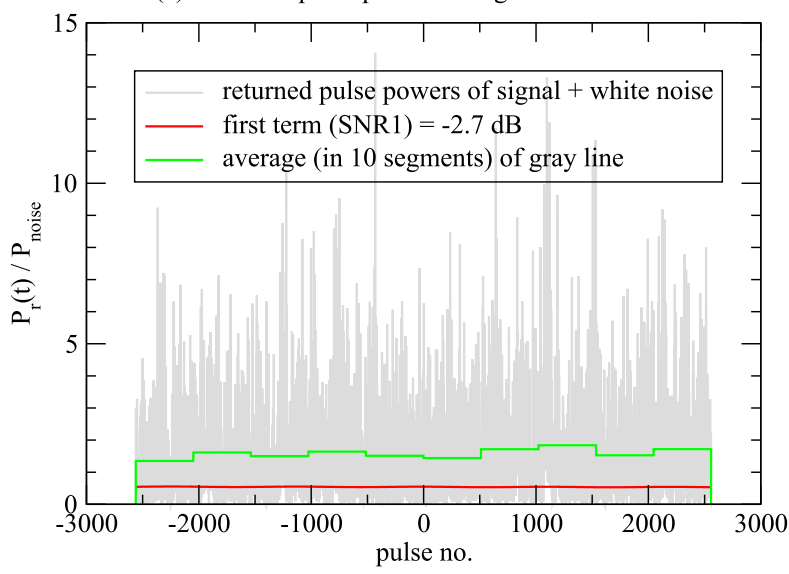

(c) Received pulse powers of signal + white noise

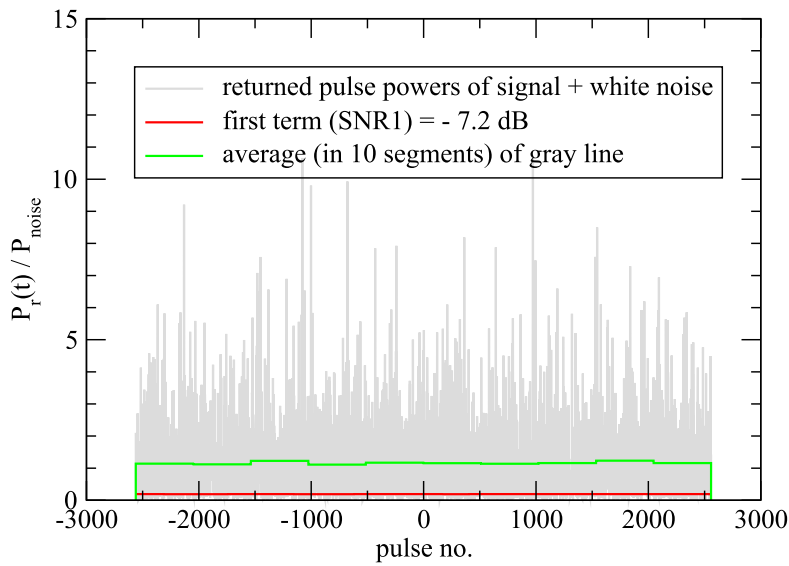

(b) Average of 10 doppler spectra of signal + white noise

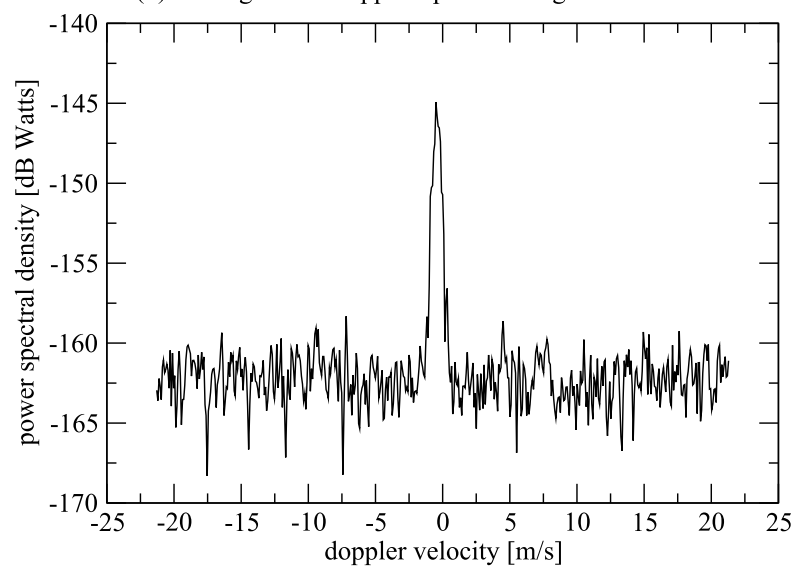

(d) Average of 10 doppler spectra of signal + white noise

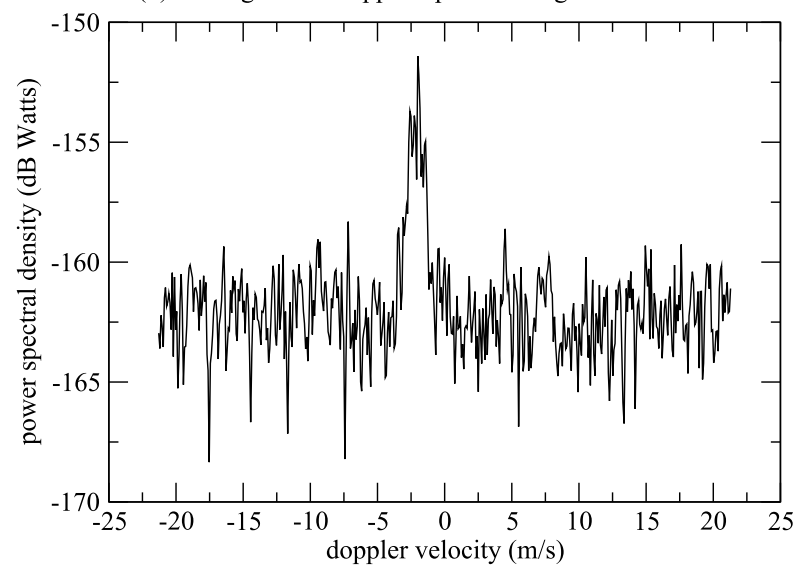

FIG. 10. Detection at low SNR1 using spectral processing. (a),(b) Resolution cell centered on $(x, y, z)=(6 \mathrm{n} \mathrm{mi},-55 \mathrm{~m},-260 \mathrm{~m})$, which is shown as the white circle to the left in Fig. 9e. (c),(d) Resolution cell centered on $(x, y, z)=(6 \mathrm{n} \mathrm{mi},-15 \mathrm{~m},-255 \mathrm{~m})$, which is shown as the white circle to the right in Fig. 9e. The radar is MIRA-35 with $\tau=0.2 \mu \mathrm{s}$ and PRF $=10 \mathrm{kHz}$. Non-IFR condition $\left(\mathrm{RH}=60 \%, T=20^{\circ} \mathrm{C}\right)$.

estimate vortex circulation, the observed velocity is multiplied by $2 \pi r$, where $r$ is the distance from the vortex center and can be determined from the location of zero radial velocity.

\section{h. Comparison of four nozzles}

Finally, Table 6 compares SNR1 reflectivity for the four nozzles listed in Table 2. The range cell for all cases is centered at $(x, y, z)=(6 \mathrm{~nm},-50 \mathrm{~m},-230 \mathrm{~m})$, which corresponds to the top of the crescent wrench in Fig. 4. IFR ambient conditions have been assumed. It is observed that the quantity $\dot{\zeta}_{\text {nozzle }}^{<}$[defined in (9)], which depends only on the droplet size distribution produced by a nozzle, provides an excellent indicator of the relative performance of different nozzles.

To provide further insight, we compute the quantity $\zeta_{x}$, which is defined to be $\zeta$ [see (8)] per unit axial length of the wake. It is calculated as a diagnostic of the droplet trajectory and size evolution and is shown in Fig. 12. The solid lines give the total value (over an entire cross section) and diagnose total evaporative loss. The dashed lines give the contribution from droplets in a neighborhood (defined in the caption) of the vortex: these curves diagnose both evaporative loss and loss by sedimentation. The ordering of $\zeta_{x}$ values (pertaining to the neighborhood of the vortex) at $x=6 \mathrm{~nm}$, also listed in the last column of Table 6 , matches the ordering of SNR1 for the different nozzles.

All statements of comparison in the following are relative to nozzle 1 and make reference to Fig. 12a. If the increased SNR1 reflectivity of nozzle 2 were due to increased volume alone, then we would get a $2.4-\mathrm{dB}$ increase in reflectivity. The actual increase is $1.03 \mathrm{~dB}$. To understand this, we first observe that nozzle 2 (solid red line) initially has a $0.7-\mathrm{dB}$ higher value of $\zeta_{x}$, less than the 2.4-dB increase in its volume flow rate. This is because nozzle 2 produces more small droplets. By $x=6 \mathrm{nmi}$ the $0.7-\mathrm{dB}$ increase has been reduced to $0.55 \mathrm{~dB}$ because the smaller droplets of nozzle 2 evaporate faster. The fact that $\zeta_{x}$ in the vicinity of the vortex 
(a) Actual radial velocity. Max. is $19.8 \mathrm{~m} / \mathrm{s}$

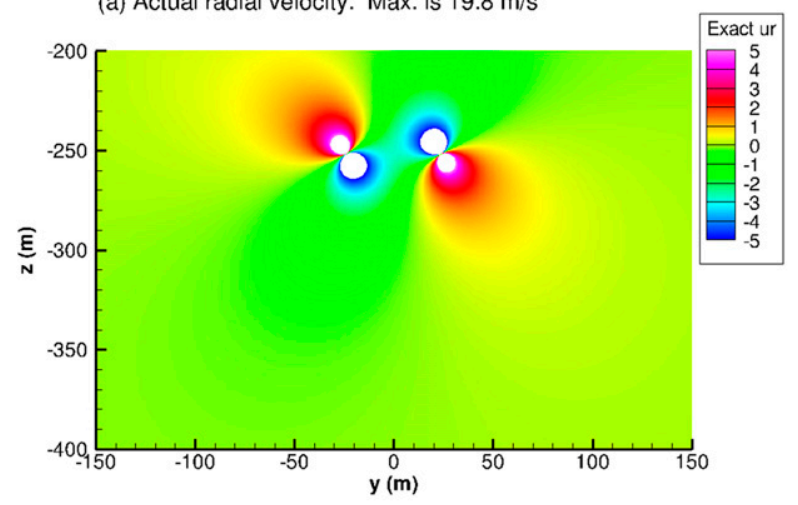

(c) DWSR 10001C, $\tau=0.2 \mu \mathrm{s}, \theta_{\mathrm{b}}=0.95 \mathrm{deg}$.

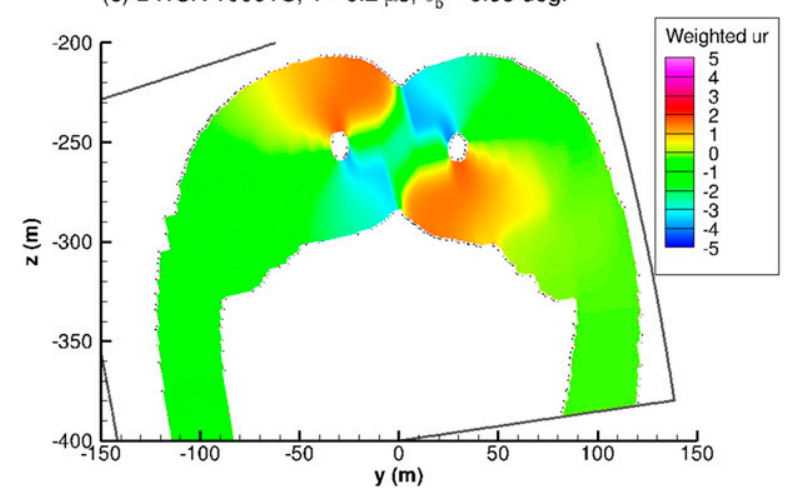

(e) MIRA-35, $\tau=0.2 \mu \mathrm{s}, \theta_{\mathrm{b}}=0.52 \mathrm{deg}$.

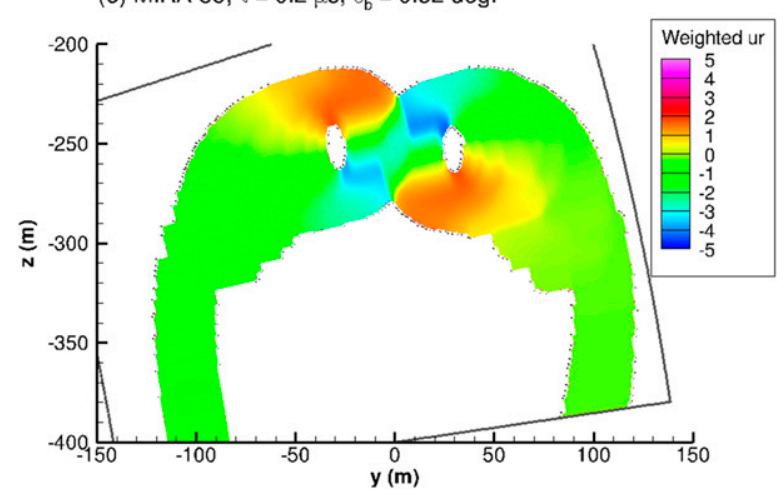

(b) DWSR $8501 \mathrm{~S}, \tau=0.4 \mu \mathrm{s}, \theta_{\mathrm{b}}=1.83 \mathrm{deg}$.

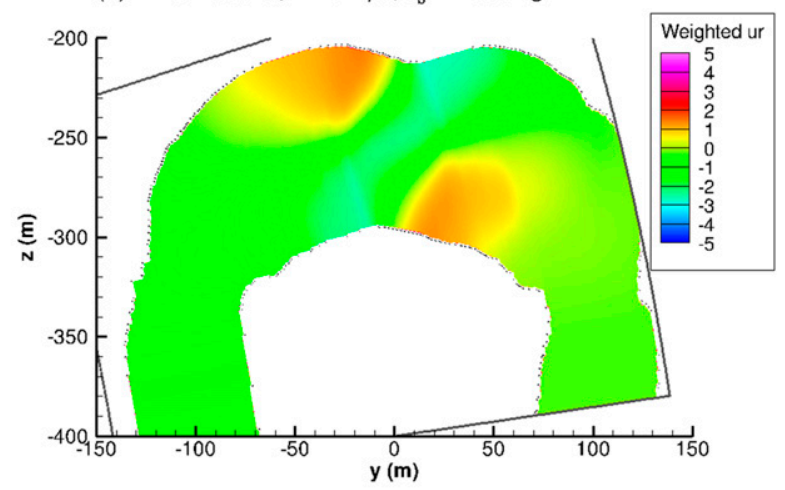

(d) DWSR 2001X, $\tau=0.2 \mu \mathrm{s}, \theta_{\mathrm{b}}=0.95 \mathrm{deg}$.

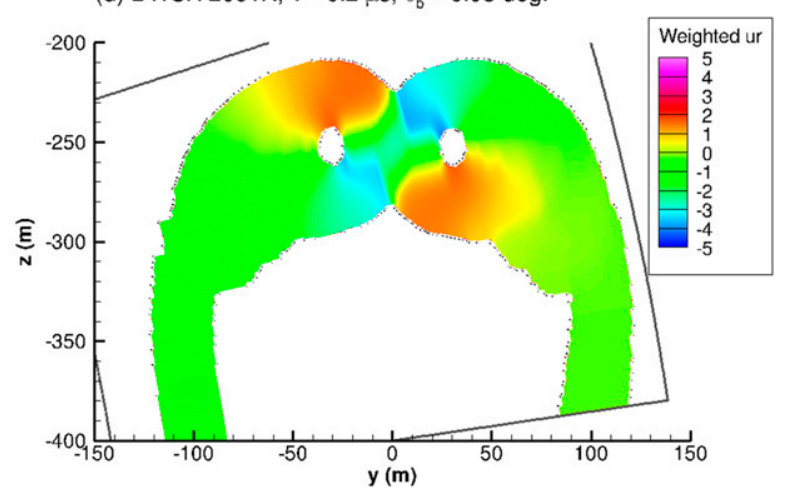

(f) $W$-SACR, $\tau=0.2 \mu \mathrm{s}, \theta_{b}=0.30 \mathrm{deg}$.

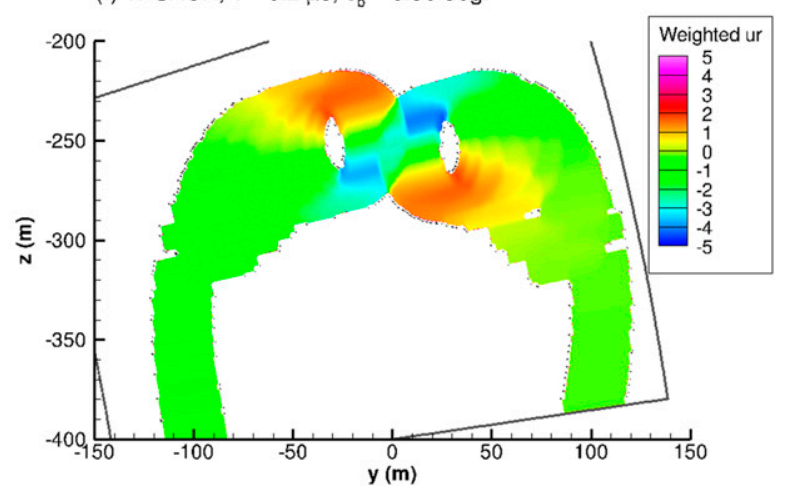

FIG. 11. Power-weighted radial velocity corresponding to Fig. 4. Only points where SNR1 $>1$ are colored. The actual radial velocity of the gas is shown in (a).

is $0.6 \mathrm{~dB}$ higher must arise from the fact that the smaller droplets of nozzle 2 have sedimented less.

Consider nozzle 3 (green curves). If its smaller reflectivity (relative to nozzle 1 ) were due to a decrease in volume, then we would expect a -0.91 drop in SNR1, which is close to what is obtained. This is understandable given that its initial $\zeta_{x}$ is very nearly the same as for nozzle 1. This is surprising given that nozzle 3 has many more smaller droplets. However, close inspection of its size distribution (green line in
Fig. 12b) shows that it also has more droplets that are very large (specifically $a>370 \mu \mathrm{m}$ ). This fact also explains the more rapid loss of $\zeta_{x}$ by sedimentation (dashed green curve in Fig. 12a) and the less rapid loss by evaporation (solid green curve). Overall, these two effects balance and the final effect that remains is due to volume decrease.

Despite its smaller flow rate, nozzle 4 has a higher initial value of $\zeta_{x}$; see the solid blue curve in Figs. 12a and $12 \mathrm{~b}$. This is because it produces more large droplets. 
TABLE 6. A comparison of SNR1 reflectivity obtained from a single range cell by using the four different nozzles listed in Table 2. A comparison with the simple a priori measure, $\dot{\zeta}_{\text {nozzle }}^{<}$, of nozzle performance is also shown. The cell center is at $(x, y, z)=$ $(6 \mathrm{n} \mathrm{mi},-50 \mathrm{~m},-230 \mathrm{~m})$. The signed values $( \pm)$ indicate values relative to nozzle 1 . MIRA-35, $\tau=0.2 \mu \mathrm{s}$, and $\theta_{\mathrm{b}}=0.52^{\circ}$.

\begin{tabular}{cccrc}
\hline \hline Nozzle No. & & & & $\frac{\zeta_{x}\left(\mathrm{~dB} \mathrm{~m}^{5}\right)}{\text { Near vortex at } x=6 \mathrm{nmi}}$ \\
\hline 1 & Gpm & SNR1 $(\mathrm{dB})$ & $\dot{\zeta}_{\text {nozzle }}^{<}\left(\mathrm{dB} \mathrm{m}^{6} \mathrm{~s}^{-1}\right)$ & -178.8 \\
2 & 3.70 & 19.15 & -157.4 & +0.6 \\
3 & 6.49 & +1.03 & -1.0 & -1.4 \\
4 & 3.00 & -1.04 & -0.3 & -1.0 \\
\hline
\end{tabular}

Unfortunately, they rapidly fall out of the wake (dashed blue curve).

\section{i. Azimuthal scanning}

Instead of having a few radars at different axial locations, all pointing at the wake at normal incidence, it would be cheaper for a single radar to azimuthally scan a wake. Figure 13 shows SNR1 for an MIRA-35 radar located at $x_{\mathrm{rad}}=4 \mathrm{nmi}$ that observes the wake at azimuthal angles of $-71.5^{\circ}, 0^{\circ}$, and $71.5^{\circ}$ measured clockwise from normal incidence. Note that the results are presented in the crosssectional plane of the aircraft rather than in the scanning plane. For these angles, the wake center is located at $x=2$, 4 , and $6 \mathrm{nmi}$ behind the aircraft, respectively. The wake is detectable at all three angles, although marginally so for the most downstream-scanning plane.

\section{Concluding remarks}

It was proposed that spraying a small amount of water into the vortex wake of a heavy aircraft during landing can make the wake visible to existing weather/ cloud radars and thereby aid air traffic controllers in selecting appropriate aircraft separations. This approach could also be used for wake vortex studies of aircraft.

Simulations of the radar reflectivity of the spray trail were performed for existing weather/cloud radars. For ambient humidity at the lower end of values typical for IFR conditions, the results showed that that good signal-to-noise (SNR) ratios (averaged over many pulses) are obtained at distances behind the aircraft of up to $6 \mathrm{~nm}$, the largest that would be contemplated given existing wake separations used in air traffic control. For the case most studied here, the amount of water spray was $3 \mathrm{gal}(\mathrm{n} \mathrm{mi})^{-1}$ of wake that needs to be detected. A currently available nozzle used for agricultural spraying can be used. A doubling of volume by doubling the number of nozzles gives a proportional increase in SNR. For a case of average humidity, evaporation was severe and pulse-averaged SNR values dropped below unity. However, since the pulse returns of the wake remained statistically stationary for 1-6s (depending on the radar), it was shown that the signal-to-noise ratio can be increased to detectable levels by spectral (Doppler) processing and averaging Doppler spectra for consecutive time segments. This would require greater dwell time for each direction at which the radar is pointed. Ultimately, selecting the dwell time for a given situation will be a trade-off between quickly completing a scan of a wake cross section and increasing SNR.

\section{a. Suggested future work}

(i) As an airplane nears the touchdown point, flaps are deflected at increasing angles. The presence of flap vortices should be included in future analysis.

(ii) The present work has ignored space-time fluctuations of the air velocity field. They will arise from the direct effect of atmospheric turbulence and from vortex core waviness induced by atmospheric turbulence, and further amplified by vortex core instabilities. Velocity fluctuations will disperse the spray trail, and if this happens on the scale of the pulse width or beamwidth, then reflectivity will be reduced. This effect should be studied in subsequent work.

(iii) There are two fluid mechanical issues that we believe are of lesser importance to the proposed concept than those listed above. They are unlikely to be resolved soon, but they should be kept in mind and considered to the extent possible. These issues are the growth of the vortex core size due to small-scale turbulence internal to or near the vortex core and the loss in circulation due to mutual annihilation.

(iv) The issue of droplet coagulation was raised by the second referee. An upper bound on the rate of coagulation is provided in section $\mathrm{C}$ of the online supplemental material with the conclusion that while coagulation should be included in future work, its effect on reflectivity is not catastrophic.

(v) The first referee raised the issue of cost. The only price we were provided and allowed to quote 
(a) $\zeta$ per axial length for one side of the wake

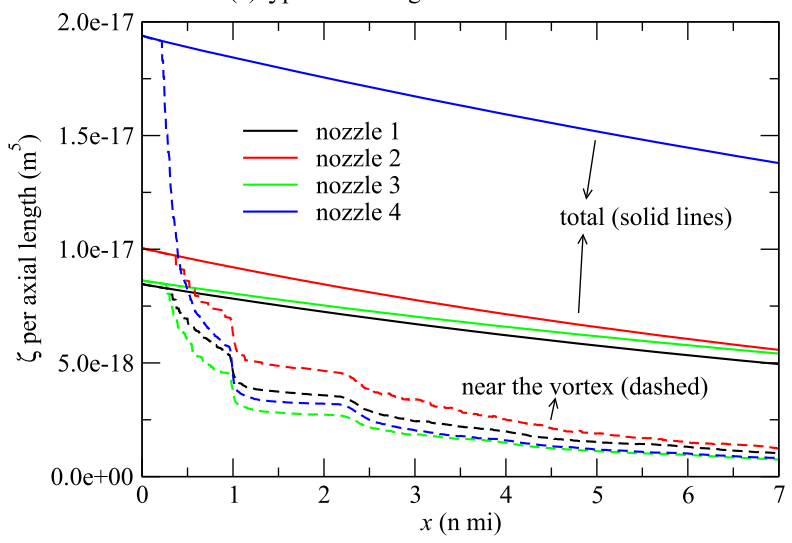

(b) Size distributions produced by the four different nozzles

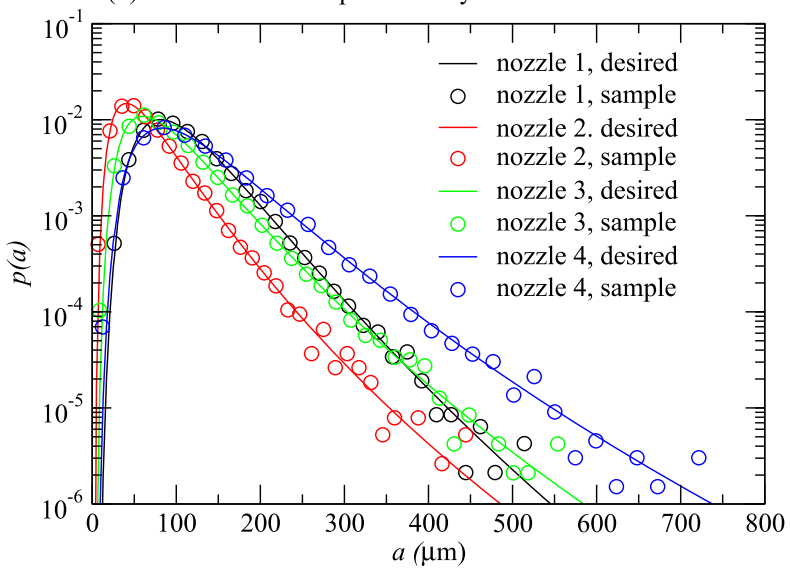

FIG. 12. (a) Term $\zeta_{x}$ for a droplet trail on one side of the aircraft. "Near the vortex" curves (dashed) were obtained by considering only those droplets that obey $|y|<60 \mathrm{~m}$ and $\left|z-z_{\text {vort }}\right|<35 \mathrm{~m}$, with $z_{\text {vort }}$ being the height of the vortex center. IFR ambient humidity and temperature were assumed. (b) Droplet size distributions produced by the four nozzles. Shown are the exact lognormal distribution (solid lines) and the distribution for each sample of 27000 droplets injected into the wake (symbols).

is that of MIRA-35, which is about EUR 0.5 million. The most expensive items in a radar system are the antenna pedestal and the power source; their price scales with size and power, respectively. We begin by excluding $\mathrm{S}$ - and $\mathrm{C}$-bands radar relative to $\mathrm{X}$ band due to the latter's smaller antenna size and higher reflectivity for the same peak power (and therefore possibly the same cost). James Mead of ProSensing suggested that the required power could be reduced by using spectral processing together with pulse compression, using a linear FM pulse, for instance. For example, with $N_{\mathrm{FFT}}=128$ and a pulse compression factor of 10 , the required power would be reduced by a factor of 1280. Scaling the peak powers in Table 4 by this factor suggests that a solid-state power amplifier (SSPA) with $150 \mathrm{~W}$ of peak power could then be used at X band or $25 \mathrm{~W}$ at Ka band. The cost of an SSPA increases with frequency; however, this might be offset by the reduced cost of the antenna pedestal with increasing frequency. One advantage of an SSPA is its high-duty cycle, which allows high pulse rates and therefore coherent processing to increase SNR. This assumes that the CPU can keep up with the data rate. This and other ideas for cost reduction should be investigated.

(vi) Consideration should be given in the future to realtime scanning and processing. For instance, how long it would take for a single radar to complete a scan of all the approach paths?

(vii) Airborne weather radar, which usually operates in the X band, was enhanced in the 1990s to detect wind shear. Section $\mathrm{E}$ in the online supplemental material shows that in order to detect a spray trail, the sensitivity of such radars needs to be improved by between 26 and $40 \mathrm{~dB}$.

\section{b. Application notes}

(i) For the purposes of simulation, we generated a spray trail that was $7 \mathrm{nmi}$ long. In practice, to reduce the volume of water, spray could be released only at a few axial locations where a detection would be performed. For each detection location, the length of the trail would need to be a few beam widths long and the release location would have to account for any head/tail wind. A trail segment that is three full beam widths long would be $0.06 \mathrm{nmi}$ long and this would require only $0.17 \mathrm{gal}$. This value assumes that $\theta_{b}=1^{\circ}$, range $=1 \mathrm{~km}$, and a flow rate of $3 \mathrm{gal}(\mathrm{n} \mathrm{mi})^{-1}$ (counting both sides of the airplane). Hence, there is considerable room for increasing water volume, and therefore signal-to-noise ratio. The main difficulty is that for the nozzles presently considered, more than one would be required for an increased flow rate. A better solution might be to design a spray head containing several nozzles.

(ii) Short trail segments would be difficult for the radar to find, particularly in the presence of wind drift and other perturbations. A continuous trail might therefore be required. In this case the total volume of water would be $18 \mathrm{gal}$ for a $6 \mathrm{nmi}$ long trail; this introduces a $150-1 \mathrm{~b}$ weight penalty. The cost of this would have to be traded off against gains in airport capacity. 
(a) Azimuth $=-71.5 \mathrm{deg}$. MIRA-35, $\tau=0.2 \mu \mathrm{s}, \theta_{\mathrm{b}}=0.52 \mathrm{deg}$.

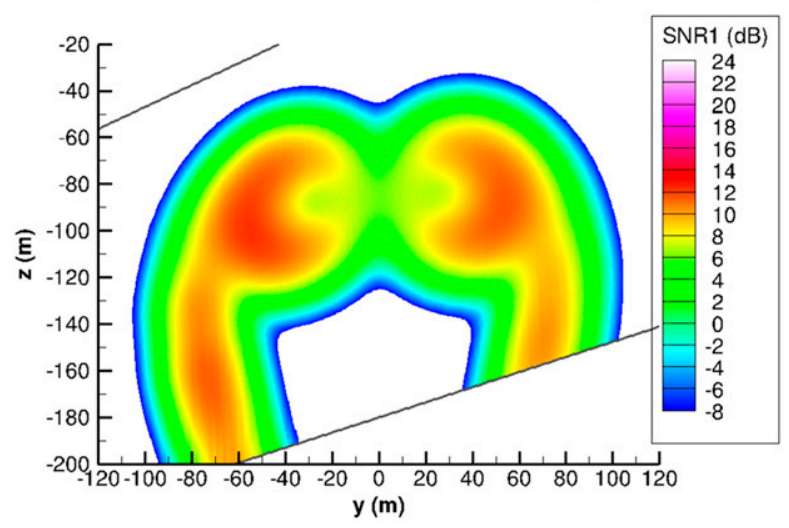

(c) Azimuth $=71.5 \mathrm{deg}$. MIRA-35, $\tau=0.2 \mu \mathrm{s}, \theta_{\mathrm{b}}=0.52 \mathrm{deg}$.

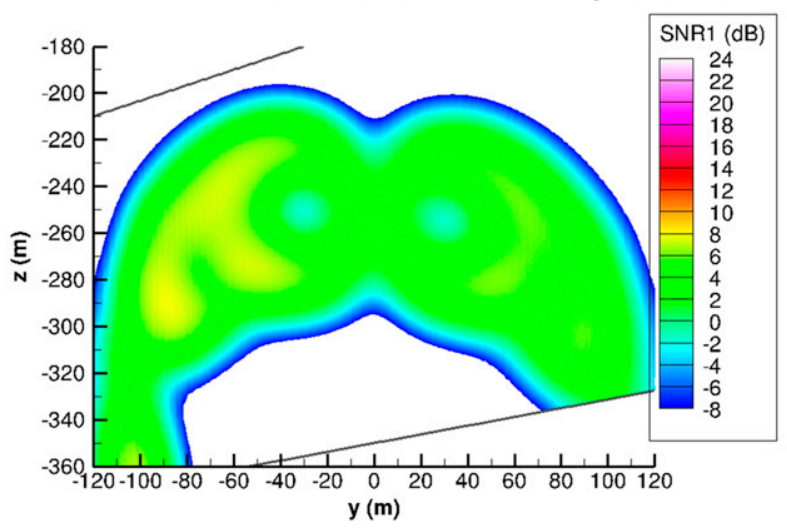

(iii) Given that spectral processing is required for detection in conditions of average humidity, it is likely that processing decisions will have to be based on humidity or on the quality of incoming returns. If the humidity is high and the quality of returns is high, then the mean velocity can be obtained from a pulse-pair estimate. If the humidity is low, then spectral processing can be turned on.

(iv) Some of the requirements of the present application are similar to those for radar imaging of tornados (French et al. 2014). This includes a smaller detection volume and the need to complete a scan faster than the vortex evolution time. Therefore, technology developed for that application could be useful here.

(v) In IFR conditions, natural precipitation (fog, mist, drizzle, or heavy rain) will be present between the radar and the wake and lead to absorption. However, at ranges of $\approx 1 \mathrm{nmi}$ envisioned for the present application, this is small.

(vi) If spraying is to be employed in very cold conditions (Denver, Colorado, comes to mind), freezing (b) Azimuth $=0$ deg. MIRA-35, $\tau=0.2 \mu \mathrm{s}, \theta_{\mathrm{b}}=0.52 \mathrm{deg}$.

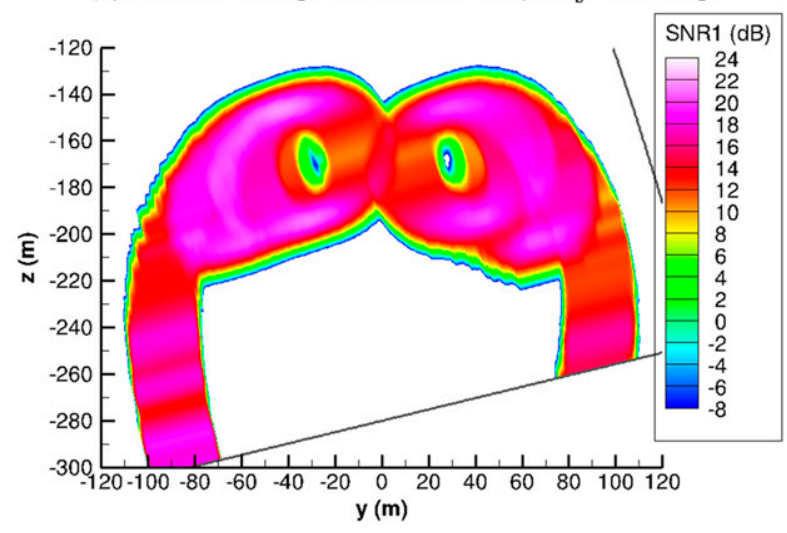

FIG. 13. SNR1 at different azimuthal angles for the MIRA-35 radar located at $x_{\text {rad }}=4 \mathrm{~nm}$. The azimuthal angle is measured clockwise relative to normal incidence. A vertical line at $y=0$ in the scanning plane is located at the following axial locations behind the aircraft for the three plots: (a) $x=2 \mathrm{~nm}$, (b) $x=4 \mathrm{~nm}$, (c) $x=6 \mathrm{~nm}$. IFR ambient conditions $\left(\mathrm{RH}=92.7 \%, T=15.2^{\circ} \mathrm{C}\right)$. Nozzle 1.

of water must obviously be prevented in the water storage and delivery system.

(vii) Several U.S. airports have approaches that are over water, which would make it difficult or impossible to implement the proposed system. Such airports include Logan International Airport (Boston, Massachusetts), San Francisco International Airport (California), St. Pete-Clearwater International Airport (Florida), New Orleans Lakefront Airport (Louisiana), and Portland International Jetport (Maine).

(viii) Dual polarization: Droplets moving relative to the air become oblate due to a higher air pressure at the front stagnation point and low pressure at $90^{\circ}$ from the front stagnation point. For falling rain droplets, this results in greater reflected power from incident waves that are horizontally versus vertically polarized (Doviak and Zrnić 1984, section 8.5.3). Most weather radars employ dual polarization to obtain more information about rainfall rate. Since, in the present case, droplets revolving around the vortices are small and their velocity relative to the air is also small, we expect that droplets will remain 
very nearly spherical. Therefore, it is not expected that dual polarization would provide additional information about the flow. However, Keränen and Chandrasekhar (2014) have suggested that dual polarization could be used for enhancing SNR. This works by exploiting coherence between signals in the horizontal and vertical channels.

(ix) Since the maximum range pertinent to the present application is much lower than for cloud and precipitation detection, the pulse repetition frequency could be increased (the maximum duty cycle of the klystron or magnetron permitting) in order to reduce the dwell time for spectral averaging.

(x) One obvious modification of existing cloud/ precipitation radar software for the present application would be a reduction in the spacing of range gates from their current values, for example, $25 \mathrm{~m}$, which is employed in Ka-band SACR (Ka-SACR) and W-SACR (Kollias et al. 2014).

(xi) Another possibility for radar placement is under the flight path. This reduces the range and therefore increases reflectivity; however, it necessitates separate radars for each approach path. With reduced range, consideration must be given to the minimum range of the radar. For the MIRA-35, the minimum range is $150 \mathrm{~m}$, which arises due to the switching delay of the transmit-receive switch. This value is constrained not only by switching technology but also by the need to protect the receiver from an unexpected strong reflector nearby, such as an airplane.

Acknowledgments. I am grateful to several individuals for sending me information when requested and sometimes more information than requested. Matthias Bauer-Pfundstein (Metek) sent me information on the MIRA-35 radar, including a detailed $\mathrm{dB} Z$ sensitivity calculation, information on signal processing, and detailed information on loss determination. It was from his $\mathrm{dB} Z$ sensitivity document that I learned that a discrete Fourier transform enhances the signal-to-noise ratio in the spectral bin of interest. John Cho (MIT Lincoln Laboratories) sent me information on the TDWR. Brad Fritz (U.S. Department of Agriculture) sent me his Excel program, which gave parameters of the droplet size distribution produced by different aerial spray nozzles at different free-stream airspeeds. $\mathrm{He}$ also experimentally measured for me the flow rate of the Davidon Tri-set nozzle. Calvin Kroes (CP Products) sent me information on nozzle orifice areas, flow rates, and exit velocities for various $\mathrm{CP}$ nozzles. Keith Vickers (Enterprise Electronics Corporation) sent me basic information on the DWSR series of radars.
Dr. James Mead (ProSensing) provided some useful cost reduction suggestions. I am grateful to Alan Wray (NASA Ames Research Center) for useful discussions. I am grateful to Jasim Ahmed and Alan Wray for performing the internal review and to the external reviewers for their useful suggestions.

\section{REFERENCES}

Apte, S., M. Gorokhovski, and P. Moin, 2003: LES of atomizing spray with stochastic modeling of secondary breakup. Int. J. Multiphase Flow, 29, 1503-1522, doi:10.1016/S0301-9322(03)00111-3.

Barbagallo, J., 2014: Aircraft wake turbulence. Federal Aviation Administration Advisory Circular 90-23G, 24 pp.

Barbaresco, F., 2012: Radar/lidar sensors for wind \& wake-vortex monitoring on airport: First results of SESAR P12.2.2 XP0 trials campaign at Paris CDG airport. [Available online at http://www. wakenet.eu/fileadmin/user_upload/4th_major_WN3E-Workshop/ presentations/WN3E_2_08_Barbaresco_SESAR12.2.2.pdf.]

_ J. Wasselin, A. Jeantet, and U. Meier, 2008: Wake vortex profiling by Doppler X-band radar: Orly trials at initial takeoff \& ILS interception critical areas. Proc. 2008 Radar Conf., Rome, Italy, IEEE, 6 pp., doi:10.1109/RADAR.2008.4721113. , P. Brovelli, P. Currier, O. Garouste, M. Klein, P. Juge, Y. Ricci, and J. Schneider, 2012: Radar sensors for wind \& wake-vortex monitoring on airport: First results of SESAR P12.2.2.2 XP0 trials campaign at Paris CDG airport. Proc. Seventh European Conf. on Radar in Meteorology and Hydrology (ERAD 2012), Toulouse, France, MétéoFrance, 4B.4.

— , and Coauthors, 2014: Radar 3D monitoring of wake-vortex hazards, circulation and EDR retrieval/calibration. Proc. 2014 Int. Radar Conf., Lille, France, SEE Society, WEO.3.11, doi:10.1109/RADAR.2014.7060419.

_, V. Brion, and N. Jeannin, 2016: Radar wake-vortices crosssection/Doppler signature characterisation based on simulation and field tests trials. IET Radar Sonar Navig., 10, 82-96, doi:10.1049/iet-rsn.2015.0132.

Batchelor, G. K., 1967: An Introduction to Fluid Dynamics. Cambridge Mathematical Library, Cambridge University Press, $658 \mathrm{pp}$.

Beckmann, P., 1962: Statistical distribution of the amplitude and phase of a multiply scattered field. J. Res. Natl. Bur. Stand., 66D, 231-240, doi:10.6028/jres.066D.027.

Crouch, J., G. Miller, and P. Spalart, 2001a: Active-control system for breakup of airplane trailing vortices. AIAA J., 39, 23742381, doi:10.2514/2.1244.

,-- , and 2001b: Development of an active system to break up trailing vortices behind commercial airplanes. Aero Mag., 14, 24-31.

Doviak, R., and D. Zrnić, 1984: Doppler Radar and Weather Observations. Academic Press, 458 pp.

Doyle, T., and F. McGee, 1998: Air traffic and operational data on selected U.S. airports with parallel runways. NASA Tech. Rep. CR-1998-207675, 59 pp.

French, M. M., H. B. Bluestein, I. PopStefanija, C. A. Baldi, and R. T. Bluth, 2014: Mobile, phased-array, Doppler radar observations of tornadoes at X band. Mon. Wea. Rev., 142, 10101036, doi:10.1175/MWR-D-13-00101.1.

Fritz, B., and W. Hoffmann, 2015: Update to the USDA-ARS fixed-wing spray nozzle models. Trans. ASABE, 58, 281-295, doi:10.13031/trans.58.10896. 
Gilson, W., 1992: Aircraft wake RCS measurement. MIT Lincoln Laboratory Project Rep. AAW-11, 65 pp.

Görsdorf, U., V. Lehmann, M. Bauer-Pfundstein, G. Peters, D. Vavriv, V. Vinogradov, and V. Volkov, 2015: A 35-GHz polarimetric Doppler radar for long-term observations of cloud parameters-Description of system and data processing. J. Atmos. Oceanic Technol., 32, 675-690, doi:10.1175/ JTECH-D-14-00066.1.

Haller, G., and T. Sapsis, 2008: Where do inertial particles go in fluid flows? Physica D, 237, 573-583, doi:10.1016/ j.physd.2007.09.027.

Hamazu, K., H. Hashiguchi, T. Wakayama, T. Matsuda, R. Doviak, and S. Fukao, 2003: A 35-GHz scanning Doppler radar for fog observations. J. Atmos. Oceanic Technol., 20, 972-986, doi:10.1175/ 1520-0426(2003)20<972:AGSDRF>2.0.CO;2.

Harris, F., 1978: On the use of windows for harmonic analysis with discrete Fourier transforms. Proc. IEEE, 66, 51-83, doi:10.1109/ PROC.1978.10837.

Ishimaru, A., 1978: Single Scattering and Transport Theory. Vol.1, Wave Propagation and Scattering in Random Media, Academic Press, 270 pp.

Keränen, R., and V. Chandrasekhar, 2014: Detection and estimation of radar reflectivity from weak echo of precipitation in dual-polarized weather radars. J. Atmos. Oceanic Technol., 31, 1677-1693, doi:10.1175/JTECH-D-13-00155.1.

Kollias, P., E. Clothiaux, M. Miller, B. Albrecht, G. Stephens, and T. Ackerman, 2007: Millimeter-wavelength radars: New frontier in atmospheric cloud and precipitation research. Bull. Amer. Meteor. Soc., 88, 1608-1624, doi:10.1175/ BAMS-88-10-1608.

—, N. Bharadwaj, K. Widener, I. Jo, and K. Johnson, 2014: Scanning ARM cloud radars. Part I: Operational sampling strategies. J. Atmos. Oceanic Technol., 31, 569-582, doi:10.1175/ JTECH-D-13-00044.1.

Lefebvre, A., 1989: Atomization and Sprays. Combustion, Hemisphere Publishing Corporation, $421 \mathrm{pp}$.

Li, J., X. Wang, and T. Wang, 2011: Modeling the dielectric constant distribution of wake vortices. IEEE Trans. Aerosp. Electron. Syst., 47, 820-830, doi:10.1109/TAES.2011.5751228.

—, T. Wang, L. Qu, H. Wang, and X. Wang, 2016: Circulation retrieval of wake vortex in fog with an upward-looking monostatic radar. IEEE Trans. Aerosp. Electron. Syst., 52, 169-180, doi:10.1109/TAES.2015.140901.

Liebe, H., G. Hufford, and T. Manabe, 1991: A model for the complex permittivity of water at frequencies below $1 \mathrm{THz}$. Int. J. Infrared Millimeter Waves, 12, 659-675, doi:10.1007/BF01008897.
Liu, Z., N. Jeannin, F. Vincent, and X. Wang, 2013: Modeling the radar signature of raindrops in aircraft wake vortices. J. Atmos. Oceanic Technol., 30, 470-484, doi:10.1175/JTECH-D-11-00220.1.

Lorenzetto, G., and A. Lefebvre, 1977: Measurements of drop size on a plain-jet airblast atomizer. AIAA J., 15, 1006-1010, doi:10.2514/3.60742.

National Research Council, 2008: Wake Turbulence-An Obstacle to Increased Air Traffic Capacity. National Academies Press, 102 pp., doi:10.17226/12044.

Pruppacher, H., and J. Klett, 1997: Microphysics of Clouds and Precipitation. Atmospheric and Oceanographic Sciences Library, Vol. 18, Kluwer Academic, 954 pp.

Radhakrishnan, K., and A. Hindmarsh, 1993: Description and use of LSODE, the Livermore Solver of Ordinary Differential Equations. NASA Reference Publ. RP-1327, LLNL Rep. UCRL-ID-113855, $122 \mathrm{pp}$.

Rayleigh, J. W. S., 1945: Theory of Sound. Vol. 1. Dover, 480 pp.

Sapsis, T., and G. Haller, 2010: Clustering criterion for inertial particles in two-dimensional time-periodic and three-dimensional steady flows. Chaos, 20, 017515, doi:10.1063/1.3272711.

Seliga, T., and J. Mead, 2009: Meter-scale observations of aircraft wake vortices in precipitation using a high resolution solid-state W-band radar. 34th Conf. on Radar Meteorology, Williamsburg, VA, Amer. Meteor. Soc., P10.25. [Available online at https://ams. confex.com/ams/34Radar/techprogram/paper_155796.htm.]

Shariff, K., and A. Wray, 2002: Analysis of the radar reflectivity of aircraft vortex wakes. J. Fluid Mech., 463, 121-161, doi:10.1017/ S0022112002008674.

Spalart, P., 1996: On the motion of laminar wing wakes in a stratified fluid. J. Fluid Mech., 327, 139-160, doi:10.1017/S002211209600849X

_ 1998: Airplane trailing vortices. Annu. Rev. Fluid Mech., 30, 107-138, doi:10.1146/annurev.fluid.30.1.107.

Vanhoenacker-Janvier, D., K. Djafri, and F. Babaresco, 2012: Model for the calculation of the radar cross section of wake vortices of take-off and landing airplanes. 2012 Ninth European Radar Conference (EuRAD), IEEE, 349-352.

Varga, C., J. Lasheras, and E. Hopfinger, 2003: Initial breakup of a small-diameter liquid jet by a high-speed gas stream. J. Fluid Mech., 497, 405-434, doi:10.1017/S0022112003006724.

Widener, K., N. Bharadwaj, and K. Johnson, 2012: Scanning ARM cloud radar (X/Ka/W-SACR): Handbook. Tech. Rep. DOE/ SC-ARM/TR-113, U.S. Dept. of Energy, Office of Science, Office of Biological and Environmental Research, $30 \mathrm{pp}$.

Yuter, S., 2003: Precipitation radar. Encyclopedia of Atmospheric Sciences, J. Holton, J. Pyle, and J. Curry, Eds., Academic Press, 1833-1851. 